\title{
FINITELY DEPENDENT COLORING
}

\author{
ALEXANDER E. HOLROYD ${ }^{1}$ and THOMAS M. LIGGETT ${ }^{2}$ \\ ${ }^{1}$ Microsoft Research, 1 Microsoft Way, Redmond, WA 98052, USA; \\ email: holroyd@microsoft.com \\ 2 Department of Mathematics, University of California, Los Angeles, CA 90095, USA; \\ email: tml@math.ucla.edu
}

Received 7 April 2015; accepted 10 September 2016

Dedicated to Oded Schramm, 10 December 1961 - 1 September 2008

\begin{abstract}
We prove that proper coloring distinguishes between block factors and finitely dependent stationary processes. A stochastic process is finitely dependent if variables at sufficiently wellseparated locations are independent; it is a block factor if it can be expressed as an equivariant finite-range function of independent variables. The problem of finding non-block-factor finitely dependent processes dates back to 1965. The first published example appeared in 1993, and we provide arguably the first natural examples. Schramm proved in 2008 that no stationary 1 -dependent 3 -coloring of the integers exists, and asked whether a $k$-dependent $q$-coloring exists for any $k$ and $q$. We give a complete answer by constructing a 1-dependent 4-coloring and a 2-dependent 3-coloring. Our construction is canonical and natural, yet very different from all previous schemes. In its pure form it yields precisely the two finitely dependent colorings mentioned above, and no others. The processes provide unexpected connections between extremal cases of the Lovász local lemma and descent and peak sets of random permutations. Neither coloring can be expressed as a block factor, nor as a function of a finite-state Markov chain; indeed, no stationary finitely dependent coloring can be so expressed. We deduce extensions involving $d$ dimensions and shifts of finite type; in fact, any nondegenerate shift of finite type also distinguishes between block factors and finitely dependent processes.
\end{abstract}

2010 Mathematics Subject Classification: 60G10, 05C15, 60C05

\section{Introduction}

Central to probability and ergodic theory is the notion of mixing in various forms. A stochastic process is a family of random variables indexed by a metric space, and mixing means that variables at distant locations are approximately

(c) The Author(s) 2016. This is an Open Access article, distributed under the terms of the Creative Commons Attribution licence (http://creativecommons.org/licenses/by/4.0/), which permits unrestricted re-use, distribution, and reproduction in any medium, provided the original work is properly cited. 
independent. The strongest and simplest mixing condition is finite dependence, which states that subsets of variables are independent provided they are at least some fixed distance apart. Despite the simplicity of the definition, finite dependence turns out to be rather subtle. Finitely dependent processes arise in the context of classical limit theorems [18, 19, 24, 27], renormalization of statistical physics models $[32,40]$, and the Lovász local lemma $[4,13]$, a fundamental tool of probabilistic combinatorics.

A key problem, originating from work of Ibragimov and Linnik in 1965 $[23,24]$, has been to understand the relationship between finite dependence and block factors. A block factor is a process that can be expressed as a function of an underlying family of independent random variables, where the function has finite range and commutes with the action of a transitive symmetry group. It is clear that a block factor is finitely dependent; it is natural to ask about the converse implication.

This question retains its interest and subtlety even in the simplest setting of stochastic processes indexed by the integer line. (We return to more general settings later.) We say that a stochastic process $X=\left(X_{i}\right)_{i \in \mathbb{Z}}$ is $\boldsymbol{k}$-dependent if the random sequences $\left(\ldots, X_{i-2}, X_{i-1}\right)$ and $\left(X_{i+k}, X_{i+k+1}, \ldots\right)$ are independent of each other, for each $i \in \mathbb{Z}$; if $X$ is $k$-dependent for some integer $k$ then it is finitely dependent. A process $X$ is stationary if $\left(X_{i}\right)_{i \in \mathbb{Z}}$ and $\left(X_{i+1}\right)_{i \in \mathbb{Z}}$ are equal in law. A process $X$ is an $\boldsymbol{r}$-block-factor (of an independent and identically distributed process) if for some independent and identically distributed $\left(U_{i}\right)_{i \in \mathbb{Z}}$ and some measurable $f$ we have $X_{i}=f\left(U_{i+1}, U_{i+2}, \ldots, U_{i+r}\right)$ for each $i$. (The random variables $U_{i}$ can be assumed uniform on $[0,1]$ without loss of generality.)

An $r$-block-factor is clearly stationary and $(r-1)$-dependent. Ibragimov and Linnik [23, 24] proved in 1965 that the converse implication holds for Gaussian processes, and claimed without proof that it is false in general. This question was explicitly stated as open by Götze and Hipp [16] and Janson [26]. It was not resolved until 1989, when Aaronson et al. [2] gave a family of 1-dependent processes that are not 2-block-factors. This construction is indirect and algebraic, and the authors asked for more natural examples. This question and the surrounding issues have been taken up by a number of authors $[1,7-10,15,17,19,25,27,32,35,36,41]$, and various further examples have been constructed. Highlights include an explicit 1-dependent (5-state) Markov chain that is not a 2-block-factor [1], a (hidden-Markov) 1-dependent process that is not an $r$-block-factor for any $r$ (Burton et al. [8]), and a 'perturbable' example showing that 2-block-factors are not dense in the set of 1-dependent Markov chains [35].

The constructions mentioned above are intricate, subtle and counterintuitive, but the resulting examples have the appearance of technical ones specifically 
constructed for the purpose. For instance, Borodin et al. [6] remarked in 2010: 'it appears that most "natural" one-dependent processes are two-block factors'. This issue has practical implications: several authors $[16,18,26]$ have been forced to assume a block-factor representation as an additional assumption in the study of finitely dependent processes: if natural finitely dependent processes are block factors, then there is little to be lost by such an assumption.

In this article we provide arguably the first genuinely natural finitely dependent stationary process that is not a block factor. Moreover, we establish something much stronger, which runs entirely counter to the above ideas about natural processes. Suppose that we impose any fixed system of local constraints on a stochastic process. (Formally, we require the process to belong almost surely to a shift of finite type.) Provided the constraints satisfy certain simple nondegeneracy conditions, we show that they can be satisfied by a stationary finitely dependent process, but not by any block factor. The latter negative statement follows from ideas of Ramsey theory-our main contribution is the former positive statement. Underlying this is a remarkable new stochastic process that is natural and canonical, yet apparently quite different from all previously studied classes of stochastic processes. It has many surprising properties that hint at a deeper theory. In particular, certain marginal projections provide unexpected links between known processes involving descent and peak sets of random permutations, Dyck words, and extremal cases of the Lovász local lemma.

Proper coloring is a canonical choice of local constraint, which turns out to be the key to the general case. We call a stochastic process $X=\left(X_{i}\right)_{i \in \mathbb{Z}}$ a $\boldsymbol{q}$-coloring (of $\mathbb{Z}$ ) if each $X_{i}$ takes values in $\{1, \ldots, q\}$, and almost surely we have $X_{i} \neq X_{i+1}$ for all $i \in \mathbb{Z}$. For which $k$ and $q$ does there exist a stationary $k$-dependent $q$-coloring of $\mathbb{Z}$ ? This question arose from discussions between Itai Benjamini, Alexander Holroyd and Benjamin Weiss in early 2008. In addition to its implications in relation to block factors, it is a formulation of the very natural question: do local constraints demand global organization? It can also be seen as a question about spontaneous symmetry breaking. Oded Schramm proved a negative answer in the first nontrivial case: there is no stationary 1-dependent 3-coloring. The proof appears in [22]; we will give a different proof, which provides some further information. It is very natural to expect that no stationary $k$-dependent $q$-coloring exists for any $k$ and $q$. (Indeed, Schramm conjectured this.) However, we prove the following.

THEOREM 1. There exist a stationary 1-dependent 4-coloring of $\mathbb{Z}$, and a stationary 2-dependent 3-coloring of $\mathbb{Z}$.

On the other hand, we have the following. 
PROPOSITION 2. No r-block-factor $q$-coloring exists, for any $r$ and $q$.

Theorem 1 and Proposition 2 together provide perhaps the cleanest answer one could hope for to the question raised by Ibragimov and Linnik:

Coloring can be done by a stationary 1-dependent process, but not by a block factor.

Moreover, since it is easily seen that no stationary finitely dependent 2-coloring exists, Schramm's impossibility result and Theorem 1 together provide a complete answer to the above question about $k$-dependent $q$-colorings. In fact, there is a canonical construction that gives precisely the two required cases $(k, q)=(1,4),(2,3)$ in Theorem 1 , and no others. To our knowledge, Theorem 1 also provides the first stationary finitely dependent non-block-factor that is symmetric under permutations of the symbols, and the first stationary 1-dependent process that is not hidden-Markov. (See below for details.)

We do not claim Proposition 2 as new, although it does not appear to be particularly well known in this form. An essentially equivalent result appears in [38] (in a stronger, quantitative form, stated in rather different terms motivated by applications in distributed computing, and building on earlier work in [33]). Further extensions and applications appear in [3, 22]. For the reader's convenience we provide a simple proof of Proposition 2.

Given the prominence of Markov chains in the literature on finitely dependent processes, it is natural to ask whether our colorings are Markov. They are not, and much more can be said. We call a stationary process $X$ hidden-Markov if there exists a stationary Markov chain $M=\left(M_{i}\right)_{i \in \mathbb{Z}}$ on a finite-state space, and a deterministic function $f$, such that $X_{i}=f\left(M_{i}\right)$ for all $i$. (In contrast with the definition of block factors, here finiteness of the state space is important: if we were to allow an uncountable state space then any stationary process $X$ could be represented this way, by taking $M_{i}=\left(\ldots, X_{i-1}, X_{i}\right)$.) Note that hidden-Markov processes include $m$-step Markov processes, as well as Gibbs measures with local interactions. The following is a previously unpublished result of Schramm, of which we present a proof.

PRoposition 3 (Schramm). No hidden-Markov finitely dependent q-coloring exists, for any $q$.

In particular, our 4-coloring provides a partial answer to a question of de Valk [10, Problem 8], who asked whether every 1-dependent process is a function of a Markov chain: the answer is no for finite-state chains. (The case of countable state spaces remains open.) 
As mentioned earlier, the colorings of Theorem 1 have many remarkable properties, which hint at some deeper structure. We strongly believe that the stationary 1-dependent 4-coloring is unique. The next result gives some of these properties, and also provides a small step toward uniqueness. Let $\mathbf{1}[\cdot]$ denote an indicator function.

TheOREM 4. The stationary 1-dependent 4-coloring $X$ and 2-dependent 3coloring $Y$ of Theorem 1 can be chosen to have the following additional properties.

(i) The processes are reversible, and symmetric under permutations of the colors, that is $X$ is equal in law to $\left(X_{-i}\right)_{i \in \mathbb{Z}}$, and to $\left(\sigma\left(X_{i}\right)\right)_{i \in \mathbb{Z}}$ for any $\sigma \in S_{4}$, and similarly for $Y$ and $\sigma \in S_{3}$.

(ii) The process $\left(\mathbf{1}\left[X_{i}=1\right]\right)_{i \in \mathbb{Z}}$ is equal in law to $\left(\mathbf{1}\left[B_{i}>B_{i+1}\right]\right)_{i \in \mathbb{Z} \text {, where }}$ $\left(B_{i}\right)_{i \in \mathbb{Z}}$ are independent and identically distributed taking values 0,1 with equal probabilities.

(iii) The process $\left(\mathbf{1}\left[X_{i} \in\{1,2\}\right]\right)_{i \in \mathbb{Z}}$ is equal in law to $\left(\mathbf{1}\left[U_{i}>U_{i+1}\right]\right)_{i \in \mathbb{Z}}$, where $\left(U_{i}\right)_{i \in \mathbb{Z}}$ are independent and identically distributed uniform on $[0,1]$.

(iv) The process $\left(\mathbf{1}\left[Y_{i}=1\right]\right)_{i \in \mathbb{Z}}$ is equal in law to $\left(\mathbf{1}\left[U_{i-1}<U_{i}>U_{i+1}\right]\right)_{i \in \mathbb{Z}}$, where $\left(U_{i}\right)_{i \in \mathbb{Z}}$ are independent and identically distributed uniform on $[0$, $1]$.

(v) The law of $\left(Y_{1}, \ldots, Y_{n}\right)$ is the conditional law of $\left(X_{1}, \ldots, X_{n}\right)$ given that $X_{i} \neq 4$ for $i=1, \ldots, n$.

Every stationary 1-dependent 4-coloring X satisfies (ii).

The processes in (ii)-(iv) above are evidently block factors, notwithstanding Proposition 2. Many of these properties are mysterious. It is not clear why conditioning a 1-dependent 4-coloring to have no 4's should be expected to give a 2-dependent process, as in (v). We have no simple explanation for the striking similarity between (iii) and (iv) (even bearing in mind (v)). It appears difficult to think of any processes satisfying the properties above, or even certain subsets of them. For example, we know of no other ergodic process $X$ that satisfies (i) and (ii), nor that satisfies the analogue of (iii) for every 2-element subset of $\{1,2,3,4\}$. It appears plausible that some such sets of properties may uniquely characterize the processes.

The processes in (ii)-(iv) have been studied extensively in other settings; (ii) is an extremal case of the Lovász local lemma in a certain sense (see $[42,43]$ and the discussion below), and (iii) and (iv) correspond to the descent sets and peak sets of random permutations (see for example [5] and references therein). 
The colorings $X$ and $Y$ can be seen as couplings of multiple copies of these processes (with special properties).

We will prove Theorem 1 by giving expressions for cylinder probabilities (that is for the probability that $\left(X_{1}, \ldots, X_{n}\right)$ takes any given value) in terms of a certain combinatorial structure. The expressions are simple but mysterious, and seem a priori very hard to guess. In the case of the 4-coloring, we will prove that the expression is equal to a very different (and more complicated) expression (an alternating sum of numbers of linear extensions of certain posets), which is useful for deducing certain properties including Theorem 4(iii) above. The equality of the two expressions also implies many interesting new combinatorial identities. We in fact started with the more complicated expression (which was guessed by considering the constraints imposed on a 4-coloring by 1-dependence), but were unable to prove its nonnegativity directly. We were led to the simple expression by searching for recursions satisfied by the complicated one.

We now consider generalizations to higher dimensions, and to general systems of local constraints (as mentioned earlier). Firstly, let $G=(V, E)$ be a graph. A stochastic process $X=\left(X_{v}\right)_{v \in V}$ indexed by the vertexes is called a $\boldsymbol{q}$-coloring if each $X_{v}$ takes values in $\{1, \ldots, q\}$ and almost surely $X_{u} \neq X_{v}$ whenever $u$ and $v$ are neighbors. It is $\boldsymbol{k}$-dependent if its restrictions to two subsets of $V$ are independent whenever the subsets are at graph distance greater than $k$ from each other. The hypercubic lattice is the graph with vertex set $\mathbb{Z}^{d}$ and an edge between $u$ and $v$ whenever $\|u-v\|_{1}=1$; the graph itself is also denoted $\mathbb{Z}^{d}$. A process on $\mathbb{Z}^{d}$ is stationary if it is invariant in law under all translations of $\mathbb{Z}^{d}$.

COROllary 5. Let $d \geqslant 2$. There exist integers $q=q(d)$ and $k=k(d)$ such that:

(i) there exists a stationary 1-dependent $q$-coloring of $\mathbb{Z}^{d}$;

(ii) there exists a stationary $k$-dependent 4 -coloring of $\mathbb{Z}^{d}$.

No stationary $k$-dependent $q$-coloring of $\mathbb{Z}^{d}$ was previously known to exist for any $k, q, d$. The proof of Corollary 5 yields explicit upper bounds on $q(d)$ and $k(d)$, but we do not expect them to be close to optimal. In particular, we can take $q(d)=4^{d}$ in (i). (See Proposition 7 below for some lower bounds.) Both assertions are consequences of Theorem 1; (i) is straightforward to deduce, while (ii) uses results of Holroyd et al. [22] that were developed for the study of finitary factor colorings. While the colorings of Corollary 5 are stationary under translations, we do not know how to make them invariant under all isometries of $\mathbb{Z}^{d}$. By another result in [22], the 4 colors in (ii) cannot be reduced to 3 for any $d \geqslant 2$. 
To describe our second extension we generalize from proper coloring to arbitrary local constraints. Write $[q]:=\{1, \ldots, q\}$. A shift of finite type on $\mathbb{Z}$ is a (deterministic) set of sequences $S \subseteq[q]^{\mathbb{Z}}$ characterized by an integer $m$ and a set $W \subseteq[q]^{m}$ of allowed local patterns as follows:

$$
S=S(q, m, W):=\left\{x \in[q]^{\mathbb{Z}}:\left(x_{i+1}, \ldots, x_{i+m}\right) \in W \forall i \in \mathbb{Z}\right\}
$$

For $w \in W$, let $T(w)$ be the set of times at which the pattern $w$ can recur, that is the set of $t \geqslant 1$ for which there exists $x \in S$ with $\left(x_{1}, \ldots, x_{m}\right)$ and $\left(x_{t+1}, \ldots\right.$, $x_{t+m}$ ) both equal to $w$. We call the shift of finite type nonlattice if there exists $w \in W$ for which $T(w)$ has greatest common divisor 1. For example, the set of all deterministic proper $q$-colorings of $\mathbb{Z}$ is a shift of finite type, and is nonlattice if and only if $q \geqslant 3$. The following is again a consequence of Theorem 1 together with results from [22].

COROLlARY 6. Let $S$ be a nonlattice shift of finite type on $\mathbb{Z}$. There exists an integer $k$ (depending on $S$ ) and a stationary $k$-dependent process $X$ such that the random sequence $X$ belongs to $S$ almost surely.

The following is a straightforward consequence of Proposition 2, proved in [22]. Let $S$ be a shift of finite type on $\mathbb{Z}$ that does not contain any constant sequence. Then there is no block factor that belongs a.s. to $S$. (In fact, under the nonlattice condition, it is shown in [22] that there is a finitary factor of an independent and identically distributed process, with tower-function decay of its coding radius, that belongs a.s. to $S$, and that this decay rate cannot be improved.) Combining this with Corollary 6 provides, as promised, an even more striking answer to the Ibragimov-Linnik question:

Any nonlattice shift of finite type on $\mathbb{Z}$ that contains no constant sequence serves to distinguish between block factors and stationary finitely dependent processes.

Returning to coloring, for any graph $G$ and any $k$ and $q$ one can ask whether there exists a $k$-dependent $q$-coloring that is invariant in law under some given group of automorphisms. The following concept leads to negative answers in some cases. A hard-core process on $G$ is a process $J=\left(J_{v}\right)_{v \in V}$ such that each $J_{v}$ takes values in $\{0,1\}$, and almost surely we do not have $J_{u}=J_{v}=1$ for adjacent vertexes $u, v$. If $X$ is a $q$-coloring of $G$ then $J_{v}:=\mathbf{1}\left[X_{v}=a\right]$ defines a hard-core process for any given color $a \in[q]$. If $X$ is $k$-dependent then so is $J$. 
We define the critical point

$$
\begin{aligned}
& p_{\mathrm{h}}=p_{\mathrm{h}}(G):=\sup \\
& \left\{p: \exists \text { a } 1 \text {-dependent hard-core process } J \text { with } \mathbb{P}\left(J_{v}=1\right)=p \forall v\right\} .
\end{aligned}
$$

It turns out that for each $p \leqslant p_{\mathrm{h}}$ there is a unique 1-dependent hard-core process with all one-vertex marginals $\mathbb{P}\left(J_{v}=1\right)$ equal to $p$. Moreover, $p_{\mathrm{h}}$ has alternative interpretations involving complex zeros of the partition function of the standard hard-core model (or lattice gas) of statistical physics, and in terms of boundary cases of the Lovász local lemma. See Section 9 and $[42,43]$ for details.

Suppose that there exists a 1-dependent $q$-coloring $X$ of $G$ in which the colors $\left(X_{v}\right)_{v \in V}$ are identically distributed. (This last condition holds in particular if the process is invariant in law under a transitive group of automorphisms.) Then the above remarks imply

$$
q \geqslant \frac{1}{p_{\mathrm{h}}}
$$

so upper bounds on $p_{\mathrm{h}}$ yield lower bounds on the number of colors needed. We illustrate the method by proving the following.

Proposition 7. Suppose that there exists a 1-dependent $q$-coloring $X$ of $G$ with $\left(X_{v}\right)_{v \in V}$ identically distributed.

(i) For $G=\mathbb{Z}^{d}$ we have $q \geqslant(d+1)^{d+1} / d^{d}$, and moreover $q \geqslant 9$ for $d=2$, and $q \geqslant 12$ for $d=3$.

(ii) For $G=T_{\Delta}$, the infinite $\Delta$-regular tree, $q \geqslant \Delta^{\Delta} /(\Delta-1)^{\Delta-1}$.

We do not know the minimum number of colors needed for a stationary 1 -dependent coloring of $\mathbb{Z}^{d}$ for any $d \geqslant 2$. On the tree $T_{\Delta}$, one may use Theorem 1 to construct 1-dependent colorings that are invariant in law under certain transitive groups of automorphisms, but again we do not know the minimum number of colors, nor whether fully automorphism-invariant colorings exist.

It is a remarkable fact that the bound (1) is tight on $\mathbb{Z}$ : we have $p_{\mathrm{h}}(\mathbb{Z})=1 / 4$, yet there exists a stationary 1-dependent 4-coloring. In other words, it is possible to couple 4 copies of the critical 1-dependent hard-core process in such a way that their supports partition $\mathbb{Z}$, while the entire process retains stationarity and 1-dependence.

One can interpret $k$-dependent processes via the language of functional analysis (see also [10]). The following is a consequence of Theorem 1. 


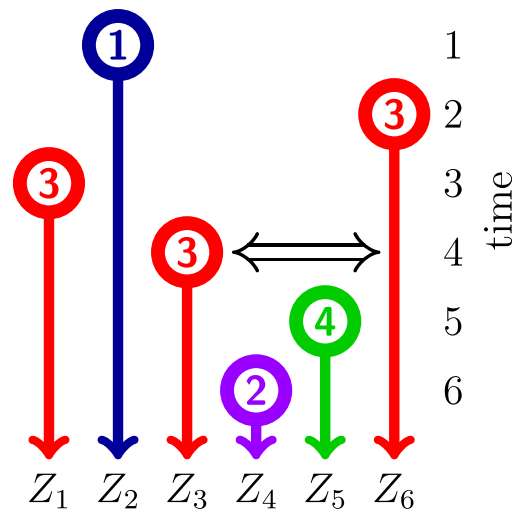

Figure 1. Construction of the process: random colors arrive at random times. In this case the coloring $\left(Z_{1}, \ldots, Z_{6}\right)$ is rejected at time 4 , because $Z_{3}$ and $Z_{6}$ are both red (color 3), and they arrive before the intervening points $Z_{4}$ and $Z_{5}$.

COROLlary 8. Let $(k, q)=(1,4)$ or $(2,3)$. There exists a real separable Hilbert space $U$ and a bounded linear operator $R: U \rightarrow U$ with the following properties. The image $R^{n} U$ is one-dimensional for all $n>k$. There is a decomposition $U=U_{1}+\cdots+U_{q}$ into mutually orthogonal closed linear subspaces, such that for each $i$, the image $R U_{i}$ is contained in the closed linear span of $\left\{U_{j}: j \neq i\right\}$.

So far as we know, Corollary 8 is new. Schramm conjectured in 2008 (motivated by colorings) that such $U$ and $R$ cannot exist for any $k$ and $q$ (even with the $U_{j}$ merely linearly independent, and without the separability restriction). A space $U$ satisfying the conditions of the corollary cannot be finite-dimensional, and by Lidskii's theorem (see for example [30, Ch. 30]), $R$ cannot be of trace class. A complex Hilbert space example has been suggested by Fedja Nazarov and Serguei Denissov (personal communication).

We now give a complete probabilistic description of our two colorings of $\mathbb{Z}$, which is astonishingly simple. (However, it is not at all obvious that it works; we will prove this in the next two sections.) See Figure 1 . Let $Z=\left(Z_{1}, \ldots, Z_{n}\right)$ be a sequence of independent and identically distributed random variables taking values $1,2, \ldots, q$ with equal probabilities. Let $\sigma$ be an independent uniformly random permutation of $1, \ldots, n$, which we interpret as meaning that the symbol $Z_{i}$ arrives at time $\sigma(i)$. Let $E$ be the event that, for every time $t=1, \ldots, n$, the subsequence of $Z$ formed by those symbols that arrived up to time $t$ (ordered as in the original sequence $Z$ ) forms a proper coloring (that is no two consecutive 
elements in the subsequence are equal). Then for $q=4$ or $q=3$, the conditional law of $Z$ given $E$ equals the law of $\left(X_{1}, \ldots, X_{n}\right)$, where $X$ is, respectively, the 4-coloring or the 3-coloring of Theorem 1.

We emphasize that the cases $q=3,4$ in the above description are very special. For $q=2$ or $q \geqslant 5$, the resulting process is not $k$-dependent for any $k$.

In a follow-up article [21] by the current authors, we use a more elaborate method inspired by the construction above to obtain for all $q \geqslant 5$ a stationary 1-dependent $q$-coloring of $\mathbb{Z}$ that is symmetric under permutations the colors (as in Theorem 4(i)). Besides these examples and straightforward embellishments of them, no other stationary finitely dependent colorings of $\mathbb{Z}$ are known.

In another article [20] by one of the current authors, the above construction is modified to obtain a probabilistic construction of the 4-coloring on the whole of $\mathbb{Z}$. (More precisely, the process is expressed as a finitary factor of an independent and identically distributed process; however, the approach fails for the 3-coloring.) One complication is that, while the laws of colorings $\left(X_{1}, \ldots, X_{n}\right)$ are consistent between different intervals (as required to obtain an extension to $\mathbb{Z}$ ), the accompanying random permutations (after conditioning) are not consistent.

The article [22] deals with the closely related issue of coloring $\mathbb{Z}^{d}$ by a finitary factor of an independent and identically distributed process; that is, a deterministic function that commutes with translations in which the color at the origin can be determined from the independent and identically distributed variables within some finite (but random and perhaps unbounded) radius. A block factor can be interpreted as a continuous map with respect to the product of discrete topologies; a finitary factor is almost everywhere continuous. Depending on the number of colors and the dimension, it turns out that the optimal tail decay of this radius is either a power law or a tower function.

The relationship between the 4-coloring and 3-coloring is puzzling. Can they be coupled in a natural way (without conditioning)? Here is one plausible approach that fails. If $X$ is a 1-dependent 4-coloring then we can obtain a 3-dependent 3-coloring $Y$ as a 3-block-factor of $X$ by eliminating color 4: take $Y_{i}$ to be $X_{i}$ unless $X_{i}=4$, in which case $Y_{i}:=\min \left(\{1,2,3\} \backslash\left\{X_{i-1}, X_{i+1}\right\}\right)$. It is natural to try to get a 2-dependent 3-coloring as a 2-block-factor of $X$, but this is impossible - this amounts to the fact that the Kautz graph with vertexes $V=\left\{(a, b) \in\{1,2,3,4\}^{2}: a \neq b\right\}$ and (undirected) edges $E=\{((a, b)$, $(b, c)):(a, b),(b, c) \in V\}$ is not 3-colorable.

Coloring, finite dependence, and block factors have applications in computer science (see for example [33, 38]). For example, colors may represent update schedules or communication frequencies for machines in a network; adjacent machines are not permitted to conflict with each other. Finite dependence implies 
privacy or security benefits: an adversary who gains knowledge of some colors learns nothing about the others, except within some fixed distance. A block factor (or, more generally, a finitary factor $[20,22]$ ) has the interpretation that colors can be computed by the machines in a distributed fashion, based on randomness generated locally together with local communication.

The article is organized as follows. In Section 2, we introduce a combinatorial structure on which our processes are based. In Section 3, we deduce Theorem 1 and Theorem 4(i,v). Sections 4-9 can largely be read independently of each other. In Sections 4 and 5, we give proofs of Propositions 2 and 3 respectively, the latter using the Hilbert space interpretation that also gives Corollary 8. In Section 6, we prove Theorem 4(ii, iv) together with the stronger assertion that every 1-dependent 4-coloring has the former property, and we give a new proof of Schramm's result that no 1-dependent 3-coloring exists. In Section 7, we provide the alternative expression for the cylinder probabilities, and deduce Theorem 4(iii). Section 8 contains the proofs of Corollaries 5 and 6, and in Section 9 we discuss hard-core processes and prove Proposition 7 . We conclude the article with a list of open problems.

\section{Buildings}

In this section, we introduce the combinatorial object on which our construction is based. We deduce some striking properties, although the real magic will happen when we interpret them probabilistically.

A word is a finite sequence $x=\left(x_{1}, x_{2}, \ldots, x_{n}\right) \in \mathbb{Z}^{n}$, which we sometimes abbreviate to $x_{1} x_{2} \cdots x_{n}$. The word $x$ is a proper coloring if $x_{i} \neq x_{i+1}$ for all $1 \leqslant i<n$. For a word $x \in \mathbb{Z}^{n}$ and a symbol $a \in \mathbb{Z}$ we denote the concatenation as $x a=\left(x_{1}, \ldots, x_{n}, a\right)$, and so forth. We write $\widehat{x}_{i}:=x_{1} \cdots x_{i-1} x_{i+1} \cdots x_{n}$ for $x$ with the $i$ th symbol removed.

Let $S_{n}$ be the symmetric group of all permutations of $1, \ldots, n$. Let $x \in \mathbb{Z}^{n}$ be a word, and let $\sigma \in S_{n}$ be a permutation. We interpret $\sigma$ as meaning that the symbol $x_{i}$ arrives at time $\sigma(i)$ (and in position $i$ ). For $t=1, \ldots, n$ we define

$$
x_{(t)}^{\sigma}:=\left(x_{i}: \sigma(i) \leqslant t\right),
$$

the subsequence of symbols that arrived by time $t$ (ordered as in $x$, not ordered by arrival times). So for example if $\sigma=(2,3,1)$ then $x_{(2)}^{\sigma}=\left(x_{1}, x_{3}\right)$. We say that $\sigma$ is a proper building of $x$ if $x_{(t)}^{\sigma}$ is a proper coloring for each $t=1$, $\ldots, n$. So the identity permutation is a proper building of the word 121 , but the permutation $(2,3,1)$ is not. Let $B(x)$ denote the number of proper buildings of $x$. The following is the key property. 
LEMMA 9. If $x$ is a proper coloring of length $n$ then

$$
B(x)=\sum_{i=1}^{n} B\left(\widehat{x}_{i}\right)
$$

Proof. This follows on considering the last arrival $\sigma^{-1}(n)$. The permutation $\sigma$ is a proper building of $x$ with $\sigma^{-1}(n)=i$ if and only if $\widehat{\sigma}_{i}$ is a proper building of $\widehat{x}_{i}$.

We deduce the following identities. Recall that $[q]:=\{1, \ldots, q\}$.

Proposition 10. Let $q \geqslant 2$ and $x \in[q]^{n}$, where $n \geqslant 0$. We have

$$
\sum_{a \in[q]} B(x a)=[n(q-2)+q] B(x) .
$$

Proposition 11. Let $x \in[q]^{m}$ and $y \in[q]^{n}$, where $m, n \geqslant 0$.

$$
\begin{aligned}
\text { If } q & =4 \text { then } \quad \sum_{a \in[q]} B(x a y)=2\left(\begin{array}{c}
m+n+2 \\
m+1
\end{array}\right) B(x) B(y) . \\
\text { If } q & =3 \text { then } \quad \sum_{a, b \in[q]} B(x a b y)=2\left(\begin{array}{c}
m+n+4 \\
m+2
\end{array}\right) B(x) B(y) .
\end{aligned}
$$

The proofs of Propositions 10 and 11 are elementary, and are very similar to each other. However, in another respect the two results are very different: Proposition 11 says something special about $q=3,4$ that apparently has no simple analogue for other $q$. For example, for $q \neq 4$ the ratio of $\sum_{a \in[q]} B(x a y)$ to $B(x) B(y)$ no longer depends only on the lengths of $x$ and $y$. Also see Proposition 13 at the end of this section.

COROLLARY 12. Let $q \geqslant 2$ and $n \geqslant 1$. The total number of proper buildings of all words of length $n$ is

$$
\Sigma(q, n):=\sum_{x \in[q]^{n}} B(x)=\prod_{k=1}^{n}[k(q-2)+2],
$$

which equals $2^{n},(n+2) ! / 2$, and $(n+1) ! 2^{n}$ in the cases $q=2,3,4$, respectively.

Proof. This is immediate from Proposition 10. (The last factor in the product is $(n-1)(q-2)+q=n(q-2)+2$. 
Proof of Proposition 10. We use induction on $n$. The identity is immediate when $n=0$ (so that $x$ is the empty word and $B(x)=1$ ). Suppose that $n \geqslant 1$ and that it holds for $n-1$. We can assume that $x$ is a proper coloring, otherwise both sides are 0 . By Lemma 9,

$$
\sum_{a \in[q]} B(x a)=\sum_{a \neq x_{n}}\left[\sum_{i=1}^{n} B\left(\widehat{x}_{i} a\right)+B(x)\right] .
$$

We now consider each of the terms on the right. For $i \leqslant n-1$ the inductive hypothesis gives

$$
\sum_{a \neq x_{n}} B\left(\widehat{x}_{i} a\right)=[(n-1)(q-2)+q] B\left(\widehat{x}_{i}\right),
$$

while for the case $i=n$ we have

$$
\sum_{a \neq x_{n}} B\left(\widehat{x}_{n} a\right)+B\left(\widehat{x}_{n} x_{n}\right)=[(n-1)(q-2)+q] B\left(\widehat{x}_{n}\right) .
$$

Since $\widehat{x}_{n} x_{n}=x$, and $\sum_{a \neq x_{n}} B(x)=(q-1) B(x)$, the right side of (2) therefore becomes

$$
[(n-1)(q-2)+q] \sum_{i=1}^{n} B\left(\widehat{x}_{i}\right)+(q-2) B(x),
$$

which by Lemma 9 equals $[n(q-2)+q] B(x)$.

Proof of Proposition 11, case $q=4$. We use induction. When $n=0$ the identity is precisely Proposition 10 with $q=4$, and the case $m=0$ follows by symmetry. Therefore, suppose that $m, n \geqslant 1$, and that the identity holds for all $x$ and $y$ with lengths totaling less than $m+n$. Assume that $x$ and $y$ are proper colorings, otherwise the identity holds trivially.

We consider two cases (and the crucial consequence of the assumption $q=4$ will be that they give identical results). First suppose $x_{m}=y_{1}$, and without loss of generality suppose both are equal to 1 . Lemma 9 gives

$$
\sum_{a \in[4]} B(x a y)=\sum_{a \neq 1}\left[\sum_{i=1}^{m} B\left(\widehat{x}_{i} a y\right)+B(x y)+\sum_{j=1}^{n} B\left(x a \widehat{y}_{j}\right)\right] .
$$

Considering the first of the three terms on the right, the inductive hypothesis gives for each $i$,

$$
\sum_{a \neq 1} B\left(\widehat{x}_{i} a y\right)=2\left(\begin{array}{c}
m+n+1 \\
m
\end{array}\right) B\left(\widehat{x}_{i}\right) B(y) .
$$


Similar reasoning applies to the third term, while $B(x y)=0$ since $x y$ is not a proper coloring. Therefore, using Lemma 9 again, the right side of (3) equals

$$
2\left(\begin{array}{c}
m+n+1 \\
m
\end{array}\right) B(x) B(y)+2\left(\begin{array}{c}
m+n+1 \\
m+1
\end{array}\right) B(x) B(y),
$$

which equals the right side of the claimed identity.

For the second case, suppose $x_{m} \neq y_{1}$, and say $x_{m}=1$ and $y_{1}=2$. Then

$$
\sum_{a \in[4]} B(x a y)=\sum_{a=3,4}\left[\sum_{i=1}^{m} B\left(\widehat{x}_{i} a y\right)+B(x y)+\sum_{j=1}^{n} B\left(x a \widehat{y}_{j}\right)\right] .
$$

For $i \leqslant m-1$ we have, similarly to the previous case,

$$
\sum_{a=3,4} B\left(\widehat{x}_{i} a y\right)=2\left(\begin{array}{c}
m+n+1 \\
m
\end{array}\right) B\left(\widehat{x}_{i}\right) B(y) .
$$

On the other hand, for $i=m$, the inductive hypothesis gives

$$
\begin{aligned}
\sum_{a=3,4} B\left(\widehat{x}_{m} a y\right)+B(x y) & =\sum_{a \neq 2} B\left(\widehat{x}_{m} a y\right) \\
& =2\left(\begin{array}{c}
m+n+1 \\
m
\end{array}\right) B\left(\widehat{x}_{m}\right) B(y) .
\end{aligned}
$$

The last of the three terms on the right of (5) can be treated similarly, and of course the middle term yields $\sum_{a=3,4} B(x y)=2 B(x y)$. (This is the key point where $q=4$ is used-for general $q$ we would be left with an additional term $(q-4) B(x y)$, which was not present in the first case above.) Therefore, the right side of (5) equals (4), as in the previous case.

Proof of Proposition 11, case $q=3$. The proof is similar to the $q=4$ case, and is again by induction. When $m$ or $n$ is 0 , the result follows by applying Proposition 10 (twice). Therefore, suppose $m, n \geqslant 1$ and that the result holds for all smaller $m+n$. Again we can assume $x$ and $y$ are proper.

By Lemma 9,

$$
\begin{aligned}
& \sum_{a, b \in[3]} B(x a b y) \\
& =\sum_{x_{m} \neq a \neq b \neq y_{1}}\left[\sum_{i=1}^{m} B\left(\widehat{x}_{i} a b y\right)+B(x b y)+B(x a y)+\sum_{j=1}^{n} B\left(x a b \widehat{y}_{j}\right)\right] .
\end{aligned}
$$


As in the previous proof, for $i \leqslant m-1$ the inductive hypothesis gives

$$
\sum_{x_{m} \neq a \neq b \neq y_{1}} B\left(\widehat{x}_{i} a b y\right)=2\left(\begin{array}{c}
m+n+3 \\
m+1
\end{array}\right) B\left(\widehat{x}_{i}\right) B(y) .
$$

The $i=m$ term must be combined with the next term, $B(x b y)$, and we again consider two cases.

Firstly, suppose $x_{m}=y_{1}=1$ (say). Then

$$
\begin{aligned}
& \sum_{1 \neq a \neq b \neq 1} B\left(\widehat{x}_{m} a b y\right)+\sum_{1 \neq a \neq b \neq 1} B(x b y) \\
& =\sum_{a b=23,32} B\left(\widehat{x}_{m} a b y\right)+\sum_{b=2,3} B\left(\widehat{x}_{m} 1 b y\right) \\
& =\sum_{a, b \in[3]} B\left(\widehat{x}_{m} a b y\right)=2\left(\begin{array}{c}
m+n+3 \\
m+1
\end{array}\right) B\left(\widehat{x}_{m}\right) B(y),
\end{aligned}
$$

by the inductive hypothesis.

Secondly, suppose $x_{m}=1 \neq 2=y_{1}$ (say). Then

$$
\begin{aligned}
& \sum_{1 \neq a \neq b \neq 2} B\left(\widehat{x}_{m} a b y\right)+\sum_{1 \neq a \neq b \neq 2} B(x b y) \\
& =\sum_{a b=21,23,31} B\left(\widehat{x}_{m} a b y\right)+B\left(\widehat{x}_{m} 13 y\right) \\
& =\sum_{a, b \in[3]} B\left(\widehat{x}_{m} a b y\right)=2\left(\begin{array}{c}
m+n+3 \\
m+1
\end{array}\right) B\left(\widehat{x}_{m}\right) B(y) .
\end{aligned}
$$

The third and forth terms appearing on the right of (6) can be treated symmetrically, so by Lemma 9 the entire sum becomes

$$
2\left[\left(\begin{array}{c}
m+n+3 \\
m+1
\end{array}\right)+\left(\begin{array}{c}
m+n+3 \\
m+2
\end{array}\right)\right] B(x) B(y),
$$

which equals the required expression.

The following fact is not needed for our main results, but it will imply that the $q$-color analogue of our processes is not finitely dependent for $q \notin\{3,4\}$.

PROPOSITION 13. Let $q \geqslant 2$ and $n \geqslant 0$. We have

$$
\sum_{x \in[q]^{n}}[B(1 \times 2)-B(1 \times 1)]=2 \prod_{k=1}^{n}[k(q-2)-2] .
$$


Proof. We use *'s to denote unrestricted symbols, so $B\left(a *^{n} b\right):=$ $\sum_{x \in[q]^{n}} B(a x b)$, and so forth. Let $n \geqslant 1$. By Lemma 9,

$$
B\left(1 *^{n} 1\right)=\sum_{\substack{x \in[q]^{n}: \\ 1 x 1 \text { proper }}}\left[B(x 1)+\sum_{i=1}^{n} B\left(1 \widehat{x}_{i} 1\right)+B(1 x)\right] .
$$

But, by symmetry,

$$
\sum_{\substack{x \in\left[q q^{n}: \\ 1 \times 1\right. \text { proper }}} B(x 1)=\sum_{a \neq 1} B\left(a *^{n-1} 1\right)=(q-1) B\left(1 *^{n-1} 2\right),
$$

and the term $B(1 x)$ can be treated similarly. On the other hand,

$$
\sum_{\substack{x \in[q]^{n}: \\ 1 x 1 \text { proper }}} B\left(1 \widehat{x}_{i} 1\right)=(q-2) B\left(1 *^{n-1} 1\right),
$$

since each proper coloring of the form $1 *^{n-1} 1$ arises from exactly $q-2$ proper colorings of the form $1 *^{n} 1$ by deleting the $(i+1)$ st symbol- the two neighboring colors must be distinct, so there are $q-2$ choices for the symbol between them that is deleted.

Therefore,

$$
B\left(1 *^{n} 1\right)=n(q-2) B\left(1 *^{n-1} 1\right)+2(q-1) B\left(1 *^{n-1} 2\right),
$$

and a similar argument gives

$$
B\left(1 *^{n} 2\right)=(n+2)(q-2) B\left(1 *^{n-1} 2\right)+2 B\left(1 *^{n-1} 1\right) .
$$

Subtracting yields

$$
B\left(1 *^{n} 2\right)-B\left(1 *^{n} 1\right)=(n(q-2)-2)\left[B\left(1 *^{n-1} 2\right)-B\left(1 *^{n-1} 1\right)\right],
$$

and induction finishes the proof.

\section{The colorings}

Proof of Theorem 1. Recall that $B(x)$ denotes the number of proper buildings of a word $x$. To construct the 4-coloring, we define

$$
P(x)=P_{4}(x):=\frac{B(x)}{\Sigma(4, n)}=\frac{B(x)}{(n+1) ! 2^{n}}, \quad x \in[4]^{n} .
$$


We claim that there is a stationary 1-dependent 4-coloring $X$ with cylinder probabilities given by

$$
\mathbb{P}\left[\left(X_{i+1}, \ldots, X_{i+n}\right)=x\right]=P(x), \quad i, n \in \mathbb{Z}, x \in[4]^{n} .
$$

Proposition 11 gives that for all words $x$ and $y$,

$$
\sum_{a \in[4]} P(x a y)=P(x) P(y) .
$$

Taking $y$ or $x$ to be the empty word $\emptyset$ gives respectively $\sum_{a \in[4]} P(x a)=P(x)$ and $\sum_{a \in[4]} P(a y)=P(y)$, so (9) gives a consistent family of measures. We have $P(\emptyset)=1$, and of course we have $P(x) \geqslant 0$ for all $x$. Thus by the Kolmogorov extension theorem (see for example [28, Theorem 6.16]) there exists a process $X$ satisfying (9), and (9) immediately shows that it is stationary. The process $X$ is a 4-coloring since $P(x)=0$ when $x$ is not a proper coloring, and (10) gives that it is 1 -dependent.

The construction of the stationary 2-dependent 3-coloring is essentially identical. We take

$$
P_{3}(x):=\frac{B(x)}{\Sigma(3, n)}=\frac{2 B(x)}{(n+2) !}, \quad x \in[3]^{n} .
$$

Consistency follows from Proposition 10, and 2-dependence from Proposition 11.

Proof of Theorem $4(i, v)$. The symmetry and conditioning properties are immediate from (8), (11), and the definition of proper buildings.

Via Proposition 10, the above proof in fact shows that for every $q \geqslant 2$ there is a symmetric, reversible, stationary $q$-coloring $X$ given by

$$
\mathbb{P}\left[\left(X_{i+1}, \ldots, X_{i+n}\right)=x\right]=\frac{B(x)}{\Sigma(q, n)} .
$$

It is immediate that this matches the description of the process via conditioning given in the introduction. The event $E$ that the random permutation $\sigma$ is a proper building of the random word $Z$ has probability $\Sigma(q, n) /\left(n ! q^{n}\right)$, which is $(n+1) / 2^{n}$ for $q=4$ and $\left(\begin{array}{c}n+2 \\ 2\end{array}\right) / 3^{n}$ for $q=3$.

Here is an alternative description of this process that does not involve conditioning, and that provides a practical and efficient method for exact sampling. Start with a sequence of length 1 consisting of a uniformly random element of $[q]$. At each step, insert a new color, in such a way that the sequence is 
always a proper coloring, as follows. Given that the current sequence has length $n-1$, choose one of the $n-2$ locations between two consecutive elements each with probability $(q-2) /[n(q-2)+2]$, or one of the 2 end locations each with probability $(q-1) /[n(q-2)+2]$. Then insert a color in the chosen location, chosen uniformly from among those that will still result in a proper coloring; there are $q-2$ choices at an internal location, or $q-1$ at an end. It is easily seen that the resulting sequence after $n-1$ such steps has the same law as $\left(X_{1}, \ldots, X_{n}\right)$. See $[34,37]$ for a somewhat related process.

Proposition 13 shows that for $q \notin\{3,4\}$ the process is not $k$-dependent for any $k$. Indeed, the right side of (7) is positive for all $q \geqslant 5$ and $n \geqslant 0$ (the product over $k$ begins $(q-4)(2 q-6)(3 q-8) \cdots)$, so the events $X_{i}=1$ and $X_{j}=1$ are strictly negatively correlated for $i \neq j$ when $q \geqslant 5$. (The case $q=2$ is trivial.)

\section{Block factors}

Proof of Proposition 2. Let $U_{1}, \ldots, U_{r+1}$ be independent and identically distributed random variables, and let $f: \mathbb{R}^{r} \rightarrow[q]$ be a measurable function. We claim that for all $r, q \geqslant 1$,

$$
\mathbb{P}\left[f\left(U_{1}, \ldots, U_{r}\right)=f\left(U_{2}, \ldots, U_{r+1}\right)\right]>0 .
$$

Once this is proved, the required result follows immediately.

We prove (12) by induction on $r$. For $r=1$ it is immediate, since $f\left(U_{1}\right)$ and $f\left(U_{2}\right)$ are independent and identically distributed. Assume that it holds for $r-1$ and all $q$. Now for $f: \mathbb{R}^{r} \rightarrow[q]$ define

$$
S\left(u_{1}, \ldots, u_{r-1}\right):=\left\{a \in[q]: \mathbb{P}\left[f\left(u_{1}, \ldots, u_{r-1}, U_{r}\right)=a\right]>0\right\},
$$

that is, the set of values that $f$ can take with positive probability given its first $r-1$ arguments. Since the function $S$ takes at most $2^{q}$ values, the inductive hypothesis gives

$$
\mathbb{P}\left[S\left(U_{1}, \ldots, U_{r-1}\right)=S\left(U_{2}, \ldots, U_{r}\right)\right]>0 .
$$

Moreover, since a.s. $f\left(U_{1}, \ldots, U_{r}\right) \in S\left(U_{1}, \ldots, U_{r-1}\right)$, we can find deterministic $A \subseteq[q]$ and $a \in A$ such that

$$
\mathbb{P}\left[S\left(U_{1}, \ldots, U_{r-1}\right)=S\left(U_{2}, \ldots, U_{r}\right)=A, f\left(U_{1}, \ldots, U_{r}\right)=a\right]>0 .
$$

Using the definition of $S\left(U_{2}, \ldots, U_{r}\right)$, and the fact that $U_{r+1}$ is independent of $\left(U_{1}, \ldots, U_{r}\right)$, the conditional probability that $f\left(U_{2}, \ldots, U_{r+1}\right)=a$ given the above event is positive. Thus,

$$
\mathbb{P}\left[f\left(U_{1}, \ldots, U_{r}\right)=f\left(U_{2}, \ldots, U_{r+1}\right)\right]>0 .
$$


By replacing ' $>0$ ' with ' $>\epsilon$ ' in the definition of $S$, the above proof can be made quantitative, giving that the left side of (12) is at least

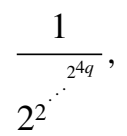

where there are $r-1$ exponentiation operations in the tower. The tower-function form of this bound is sharp. See [22] for more information.

\section{Hilbert spaces and hidden-Markov processes}

In this section, we present the Hilbert space connection that leads to Corollary 8, and from which we will also deduce Proposition 3 concerning hidden-Markov processes.

Before doing this we give the much simpler proof of a special case of Proposition 3: a stationary $k$-dependent $q$-coloring cannot itself be a Markov chain. Indeed, let $\mathbf{P}=\left(P_{a, b}\right)_{a, b \in[q]}$ be its transition matrix. Since $X_{n}$ is independent of $X_{0}$ for $n>k$, the conditional law of $X_{n}$ given $X_{0}$ is simply the stationary distribution of the Markov chain, so in particular the conditional laws of $X_{k+1}$ and $X_{k+2}$ given $X_{0}$ are identical, hence $P^{k+1}=P^{k+2}$, that is $P^{k+1}(1-P)=0$. Therefore, the eigenvalues of $P$ are precisely 0 and 1 . However, since $X$ is a proper coloring we have $P_{a, a}=0$ for all $a$, so $P$ has trace 0 , and its eigenvalues (with multiplicities) sum to 0 , a contradiction.

The proof of Proposition 3 follows a broadly similar strategy, but requires a more elaborate set-up, which also gives Corollary 8. Let $X=\left(X_{i}\right)_{i \in \mathbb{Z}}$ be a stationary process taking values in $\Omega:=[q]^{\mathbb{Z}}$, with law $\mu$. Let $L^{2}$ be the Hilbert space of real $L^{2}(\mu)$ functions on $\Omega$ (which is separable by the Stone-Weierstrass and Lusin theorems). Let $S: \Omega \rightarrow \Omega$ be the shift map given by $S(x)_{j}=x_{j-1}$, and define the shift operator $T: L^{2} \rightarrow L^{2}$ by $(T f)(x)=f\left(S^{-1}(x)\right)$. Let $A$ be the space of functions $f \in L^{2}$ that depend only on $x_{0}, x_{1}, \ldots$, and let $B$ be the space of functions $f \in L^{2}$ that depend only on $\ldots, x_{-1}, x_{0}$. Thus $T A \subseteq A$ and $T B \supseteq B$. Let $P_{B}$ denote orthogonal projection in $L^{2}$ onto $B$, or in probabilistic terms, $P_{B}(f)=\mathbb{E}\left(f \mid \ldots, X_{-1}, X_{0}\right)$. Define

$$
U:=\overline{P_{B} A}
$$

(where the bar denotes closure), and define $R$ to be the restriction

$$
R:=\left.\left(P_{B} T\right)\right|_{U} .
$$

LEMMA 14. Let $X=\left(X_{i}\right)_{i \in \mathbb{Z}}$ be a stationary process taking values in $[q]^{\mathbb{Z}}$. Define the Hilbert space $U$ and the operator $R$ as above. 
(i) We have $R U \subseteq U$.

(ii) If $X$ is $k$-dependent, then $R^{n} U$ is the space of constant functions, for all $n>k$.

(iii) If $X$ is a q-coloring, then $U$ has an orthogonal decomposition

$$
U=U_{1} \oplus \cdots \oplus U_{q}
$$

into closed linear subspaces such that $R U_{j}$ is orthogonal to $U_{j}$ for each $j$.

Proof. We first note that

$$
P_{B} T P_{B}=P_{B} T \text {. }
$$

Indeed, this is an instance of the tower property. When applied to $f \in L^{2}$, the right side yields the conditional expectation of $T f$ given $\left(X_{i}\right)_{i \leqslant 0}$, while the left side yields the conditional expectation of $T f$ given $\left(X_{i}\right)_{i \leqslant 1}$ and then $\left(X_{i}\right)_{i \leqslant 0}$.

Now suppose that $f \in A$ and $g=P_{B} f$. Then (13) gives $R g=R P_{B} f=$ $P_{B} T P_{B} f=P_{B} T f \in P_{B} A$. Thus $R$ maps $P_{B} A$ into itself. Since $R$ is continuous, the same applies to the closure $U$, establishing (i).

A similar argument to the above gives $R^{n} U \subseteq \overline{P_{B} T^{n} A}$ for every integer $n \geqslant 1$. Now if $X$ is $k$-dependent then $P_{B} T^{n} A$ is the space of constants for all $n>k$, so we obtain (ii).

Finally, let $V_{j}$ denote the space of functions in $L^{2}$ that are supported on the set of $x \in \Omega$ such that $x_{0}=j$. Let

$$
U_{j}:=\overline{P_{B}\left(V_{j} \cap A\right)} .
$$

Then $U_{j} \subseteq V_{j}$, since $P_{B} V_{j} \subseteq V_{j}$ and $V_{j}$ is closed. The spaces $V_{j}$ are mutually orthogonal, therefore so are $U_{j}$. Clearly, $A$ is the direct sum of the subspaces $V_{j} \cap A$, and therefore $P_{B} A$ is spanned by the spaces $P_{B}\left(V_{j} \cap A\right)$. Since these are mutually orthogonal, the same applies to the closures. So $U$ is the orthogonal direct sum of the spaces $U_{j}$.

Now suppose that $X$ is a $q$-coloring; then $V_{j}$ is orthogonal to $T V_{j}$. To prove (iii) we must show that $R U_{j}$ and $U_{j}$ are orthogonal. Suppose $f, g \in U_{j}$. Then $\langle f, R g\rangle=\left\langle f, P_{B} T g\right\rangle=\left\langle P_{B} f, T g\right\rangle=\langle f, T g\rangle=0$. (Here we used that $P_{B}$ is an orthogonal projection and therefore self-adjoint, and that $f, g \in V_{j}$ so $f$ and $T g$ are orthogonal.) This proves (iii).

Proof of Corollary 8. This is immediate by Theorem 1 and Lemma 14.

To prove Proposition 3 we also need the following.

LEMMA 15. If $X$ is a hidden-Markov process then the Hilbert space $U$ defined above has finite dimension. 
Proof. Let $X$ be a function of a Markov chain $M$ with state space $S$. Consider the earlier space $L^{2}=L^{2}(\mu)$ embedded in the possibly larger space of $L^{2}(\lambda)$ functions on the probability space of $M$, where $\lambda$ is the law of $M$, and where we now interpret a function $f \in L^{2}(\mu)$ as the random variable $f(X)$. Let $C$ be the space of random variables in $L^{2}(\lambda)$ that depend only on $\ldots, M_{-1}, M_{0}$, and let $P_{C}$ denote orthogonal projection onto $C$. Since $X_{i}$ is a function of $M_{i}$ we have $B \subseteq C$, and therefore $U=\overline{P_{B} A}=\overline{P_{B} P_{C} A}$, so it suffices to prove that $P_{C} A$ is finite-dimensional. Let $f \in A$. Then

$$
P_{C} f=\mathbb{E}\left(f \mid \ldots, M_{-1}, M_{0}\right)=\mathbb{E}\left(f \mid M_{0}\right),
$$

by the Markov property. But the latter depends only on $M_{0}$, so it is in the linear span of the functions $\left\{\mathbf{1}\left[M_{0}=s\right]: s \in S\right\}$. Thus $\operatorname{dim}\left(P_{C} A\right) \leqslant|S|$.

Proof of Proposition 3. Apply Lemmas 14 and 15. Since $U$ is finite-dimensional, choose an orthonormal basis $e_{1}, \ldots, e_{d}$ that comprises orthonormal bases for each $U_{j}$. Since $R e_{i}$ is orthogonal to $e_{i}$ for each $i$ we have $\operatorname{trace}(R)=0$. But Lemma 14(ii) implies that $R$ has exactly one nonzero eigenvalue, a contradiction.

Hilbert space representations of $k$-dependent processes were also explored in [10]. We briefly discuss the connection with the above approach. It is shown in [10] that if $X$ is a stationary $k$-dependent $[q]$-valued stochastic process, there exist a Hilbert subspace $H$ of $L^{2}$ and bounded linear operators $A_{1}, \ldots, A_{q}$ on $H$ that encapsulate the cylinder probabilities of $X$ via

$$
P\left(\left(X_{1}, \ldots, X_{n}\right)=x\right)=\left\langle A_{x_{1}} \cdots A_{x_{n}} \mathbf{1}, \mathbf{1}\right\rangle
$$

with the subsidiary conditions

$$
\begin{gathered}
\left(A_{1}+\cdots+A_{q}\right)^{k} h=\langle h, \mathbf{1}\rangle \mathbf{1}, \quad h \in H, \\
\left(A_{1}+\cdots+A_{q}\right) \mathbf{1}=\mathbf{1}, \\
\left(A_{1}^{*}+\cdots+A_{q}^{*}\right) \mathbf{1}=\mathbf{1},
\end{gathered}
$$

where $\mathbf{1}$ is the function that is identically 1 . The subspace $H$ is not given explicitly in [10], though the operators $A_{i}$ are. The construction above provides an explicit choice:

$$
H=\overline{R U}, \quad A_{i}=P_{H} I_{i} T,
$$

where $I_{i}=\mathbf{1}\left[X_{1}=i\right]$. (These $A_{i}$ 's are the same as in [10].) To check (14), for example, take $h \in H$ and note that, since $H \subseteq B$, we have $P_{B} T h=$ $R h \in R^{2} U \subseteq H$, so that $P_{H} T h=R h$. Iterating gives $\left(P_{H} T\right)^{n} h=R^{n} h$ for $n \geqslant 1$. Since $A_{1}+\cdots+A_{q}=P_{H} T$, Lemma 14(ii) gives (14). 


\section{One-color marginals}

Theorem 4(ii) is a consequence of the following more general result that in any 1-dependent coloring, the set of locations of a single color has a simple structure.

PROPOSITION 16. Suppose that $\left(X_{i}\right)_{i \in \mathbb{Z}}$ is a stationary 1-dependent $q$-coloring. Suppose $p:=\mathbb{P}\left(X_{0}=1\right)>0$. Then the process $J$ defined by $J_{i}:=\mathbf{1}\left[X_{i}=\right.$ $1]$ is a renewal process, and its renewal time $T$ (the number of steps between consecutive 1's) has probability generating function

$$
G(s):=\mathbb{E} s^{T}=\frac{p s^{2}}{1-s+p s^{2}} .
$$

The fact that $J$ is a renewal process is due to Fuxi Zhang. We are grateful for her permission to include it.

Proof of Proposition 16. To prove that $J$ is a renewal process we must check that $\left(J_{i}\right)_{i<0}$ and $\left(J_{i}\right)_{i>0}$ are conditionally independent given $J_{0}=1$. Since $X$ is a coloring, $J_{0}=1$ implies $J_{-1}=J_{1}=0$. For a string $u \in\{0,1, *\}^{n}$ we write $\mathbb{P}(u):=\mathbb{P}\left(J_{i}=u_{i} \forall i\right.$ s.t. $\left.u_{i} \neq *\right)$ (so that $*$ 's denote unrestricted symbols). Let $u, v \in\{0,1\}^{n-1}$ be any binary words. Then

$$
\begin{aligned}
\mathbb{P}(u 010 v) & =\mathbb{P}(u * 1 * v) \\
& =p \mathbb{P}(u) \mathbb{P}(v) \\
& =p^{-1} \mathbb{P}(u * 1) \mathbb{P}(1 * v) \\
& =p^{-1} \mathbb{P}(u 01) \mathbb{P}(10 v)
\end{aligned}
$$

(where in the 2nd and 3rd equalities we used 1-dependence of $J$, and in the 1st and 4th we used the fact that $J$ has no consecutive 1's). Now dividing through by $p$ shows that the events $\left(J_{-n}, \ldots, J_{-1}\right)=u 0$ and $\left(J_{1}, \ldots, J_{n}\right)=0 v$ are conditionally independent given $J_{0}=1$, as required.

Turning to the renewal time distribution, we write

$$
p_{n}=\mathbb{P}\left(10^{n-1} 1\right) / p .
$$

This is the conditional probability given that we have just seen 1 of waiting $n$ steps until the next 1 , thus $\left(p_{n}\right)_{n \geqslant 1}$ is the probability mass function of the renewal time. Note that $p_{1}=0$. The probability generating function is defined by

$$
G(s):=\sum_{n \geqslant 1} p_{n} s^{n}
$$


Since $J$ is a renewal process, for any integers $k_{i}>0$ we have

$$
\mathbb{P}\left(10^{k_{1}-1} 10^{k_{2}-1} 1 \cdots 0^{k_{m}-1} 1\right)=p p_{k_{1}} p_{k_{2}} \cdots p_{k_{m}} .
$$

We claim that

$$
p\left(G(s)+G(s)^{2}+G(s)^{3}+\cdots\right)=p^{2}\left(s^{2}+s^{3}+s^{4}+\cdots\right) .
$$

To check this, observe that by (15), the coefficient of $s^{n}$ on the left side is the sum of $\mathbb{P}(1 u 1)$ over all binary strings $u$ of length $n-1$. But this is simply $\mathbb{P}\left(1 *^{n-1} 1\right)$, which equals 0 for $n=1$ (by the coloring property) and $p^{2}$ for $n \geqslant 2$ (by 1 dependence), as required for the right side.

Finally, summing the geometric series in (16) and solving gives the claimed formula for $G(s)$.

Proposition 16 yields an alternative proof of the following result of Schramm (see [22] for Schramm's original proof).

COROLlARY 17. In any stationary 1-dependent q-coloring, any given color has marginal probability at most $1 / 4$. In particular, there is no stationary 1-dependent 3-coloring.

Proof. Suppose that $p>1 / 4$. Then both singularities of $G$ (viewed as a function on the complex plane) are complex. This contradicts a theorem of Pringsheim from 1893 (see [14, Theorem IV.6] or [45, Section 7.21]): a Taylor series with nonnegative real coefficients and finite radius of convergence $R$ has a singularity at $R$. (Alternatively, one may show that $G$ has negative coefficients by expressing it in partial fractions.)

Proof of Theorem 4(ii). We prove that any stationary 1-dependent 4-coloring has property (ii), as claimed at the end of Theorem 4. By Corollary 17, each color must have marginal probability exactly $p=1 / 4$, in which case the probability generating function of the renewal time in Proposition 16 factorizes to become

$$
G(s)=\left(\frac{s}{2-s}\right)^{2} .
$$

But this is the probability generating function of the sum of two independent Geometric(1/2) random variables, which yields the claimed description of the process $J$.

One straightforward consequence of Theorem 4(ii) is that for any stationary 1-dependent 4-coloring $X$,

$$
\mathbb{P}\left(X_{1}, \ldots, X_{n} \in\{2,3,4\}\right)=\frac{n+2}{2^{n+1}} .
$$


For our 4-coloring this also follows from Corollary 12 with $q=3$ (and symmetry).

Corollary 17 and its proof reflect the fact that $q=4$ colors is in a sense a critical case for the 1-dependent coloring problem. This is one reason for our belief that the solution is unique. See Section 9 for extensions of some of these ideas to general graphs.

Finally in this section we derive the claimed description of the one-color marginal for the 3-coloring, for which we need to return to proper buildings.

Proof of Theorem 4(iv). It suffices to check that the two processes have equal probabilities of assigning 1's to every integer in a finite set $A \subset \mathbb{Z}$, since all other cylinder probabilities can be computed from these by inclusion-exclusion. Since both processes are 2-dependent and have no adjacent 1's, it is enough to do this for $A$ of the form $\{1,3, \ldots, 2 m-1\}$.

Let $P(x)=P_{3}(x)=2 B(x) /(n+2)$ ! denote the cylinder probability of the 3 -coloring for the word $x \in[3]^{n}$. We use $*$ 's to denote unrestricted symbols in [3] to be summed over, so that 2-dependence of the process says that $P(x * * y)=$ $P(x) P(y)$ for all words $x$ and $y$. Lemma 9 gives that for every proper coloring $x \in[3]^{n}$,

$$
(n+2) P(x)=\sum_{i=1}^{n} P\left(\widehat{x}_{i}\right) .
$$

Write $p_{m}:=P(1 * 1 * 1 \cdots * 1)$, where the word has $m 1$ 's and length $2 m-1$, and $p_{0}:=1$. Then,

$$
\begin{aligned}
(2 m+1) p_{m} & =P(* 1 * 1 * 1 \cdots)+P(1 * * 1 * 1 \cdots)+P(1 * 1 * * 1 \cdots)+\cdots \\
& =p_{0} p_{m-1}+p_{1} p_{m-2}+\cdots+p_{m-1} p_{0} .
\end{aligned}
$$

(The first equality requires some care: the left side does not change if we interpret each $*$ as being summed over $\{2,3\}$ instead of [3]; then we can apply (17). The words that arise from deleting a $*$ vanish, since they are not proper colorings, and in the others we may allow each $*$ to revert to its original meaning, since it is still adjacent to a 1 . For the second equality we use 2-dependence.)

We now show that the cylinder probabilities of the second process satisfy the same recurrence, whereupon induction will finish the proof. Indeed, let $q_{m}:=\mathbb{P}\left(U_{1}<U_{2}>U_{3}<\cdots>U_{2 m+1}\right)$, where the inequalities alternate, and $q_{0}:=1$. This equals the probability of the event $E$ that the elements of a uniformly random permutation $\pi$ in $S_{2 m+1}$ satisfy the same inequalities. We decompose $E$ according to the location of the maximum of $\pi$. The conditional probability of $E$ given $\pi_{2 i}=2 m+1$ is

$$
\mathbb{P}\left(\cdots<\pi_{2 i-2}>\pi_{2 i-1}\right) \mathbb{P}\left(\pi_{2 i+1}<\pi_{2 i+2}>\cdots\right)=q_{i-1} q_{m-i} .
$$




\section{Alternative formula}

In this section, we derive a different formula for the cylinder probabilities of the 1-dependent 4-coloring $X$ of $\mathbb{Z}$. It was this formula that originally convinced us that such a coloring must exist (contrary to much circumstantial evidence), since it has all the required properties, except that it appears extremely difficult to prove directly that it is nonnegative. We were led to our solution by seeking recursions satisfied by this formula, and finding the equivalent of Lemma 9 (which we then re-interpreted via buildings). Below we state the formula, after some necessary definitions. We then discuss applications and motivation before giving the proof. The basic idea is to start with a postulated law for the 1-dependent binary process $\left(\mathbf{1}\left[X_{i}=1 \text { or } 2\right]\right)_{i \in \mathbb{Z}}$, and try to build the law of $X$ around it.

We identify the 4 colors with binary strings of length 2 . It is convenient to use the binary symbols $+(=+1)$ and $-(=-1)$, and to write the strings as column vectors, so $1,2,3,4=\left(\begin{array}{l}- \\ -\end{array}\right),\left(\begin{array}{l}- \\ +\end{array}\right),\left(\begin{array}{l}+ \\ -\end{array}\right),\left(\begin{array}{l}+ \\ +\end{array}\right)$ (say; the choice of bijection is immaterial). Then a word $x \in[4]^{n}$ becomes a $2 \times n$ matrix, and we denote its rows $y, z \in\{-,+\}^{n}$ :

$$
x=\left(x_{1}, x_{2}, \ldots, x_{n}\right)=\left(\begin{array}{l}
y \\
z
\end{array}\right)=\left(\begin{array}{llll}
y_{1} & y_{2} & \cdots & y_{n} \\
z_{1} & z_{2} & \cdots & z_{n}
\end{array}\right) .
$$

Let $y \in\{-,+\}^{n}$, and let $\alpha(y)$ denote the number of permutations $\pi \in S_{n+1}$ such that $\pi_{i}<\pi_{i+1}$ if $y_{i}=+$, and $\pi_{i}>\pi_{i+1}$ if $y_{i}=-$, for each $1 \leqslant i \leqslant n$ (in other words, the number of permutations with descent set given by the locations of -'s, or the number of linear extensions of the $(n+1)$-element poset generated by these inequalities). For example,

$$
\begin{aligned}
& \text { if } \quad y=\quad+\quad-\quad+\quad+\text {. } \\
& \text { then } \alpha(y)=\left|\left\{\pi \in S_{5}: \pi_{1}<\pi_{2}>\pi_{3}<\pi_{4}<\pi_{5}\right\}\right|=9 .
\end{aligned}
$$

(See for example [39] for information about $\alpha$ ). If $\left(U_{i}\right)_{i \in \mathbb{Z}}$ are independent and identically distributed uniform on $[0,1]$ and we let $Y_{i}:=(-1)^{1\left[U_{i}>U_{i+1}\right]}$ then $\mathbb{P}\left(\left(Y_{1}, \ldots, Y_{n}\right)=y\right)=\alpha(y) /(n+1)$ !. This will be the law of $Y$, where $X=\left(\begin{array}{l}Y \\ Z\end{array}\right)$. A Dyck word of length $2 k$ is an element of $\{-,+\}^{2 k}$ comprising $k+$ 's and $k$ -'s, such that the $i$ th + precedes the $i$ th - for each $i$. A dispersed Dyck word of length $m$ is an element of $\{-, 0,+\}^{m}$ that is a concatenation of Dyck words and strings of 0's. Examples of dispersed Dyck words are +-0++--00, 000, and +-+- (but not $+0-$ ). Let $\mathrm{DD}(m)$ be the set of dispersed Dyck words of length $m$, and for $w \in \mathrm{DD}(m)$, let $|w|$ be the number of +'s in $w$. (We remark that $|\mathrm{DD}(m)|=\left(\begin{array}{c}m \\ \lfloor m / 2\rfloor\end{array}\right)$, although we will not use this. For a bijective proof, consider a 
lattice path from $\left(0, \frac{1}{2}\right)$ to $\left(m, \pm \frac{1}{2}\right)$ via steps $(1, \pm 1)$. Map steps between heights $-\frac{1}{2}$ and $\frac{1}{2}$ to 0 's, and reflect excursions below $-\frac{1}{2}$ into excursions above $\frac{1}{2}$.)

If $y \in\{-,+\}^{n}$ has $m$ intervals of constancy (or runs) and $w \in \operatorname{DD}(m-1)$, define $y_{w} \in\{-,+\}^{n}$ to be the word obtained by changing the signs of some whole runs of $y$, not including the first and last runs, in such a way that the $j$ th sign change between runs is eliminated precisely for those $j$ with $w_{j} \neq 0$. For example, with $n=15$ and $m=9$,

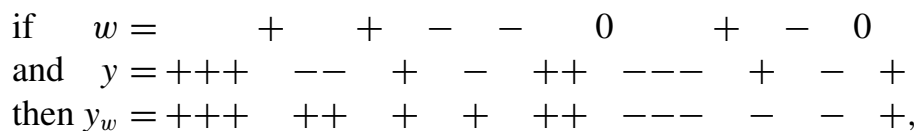

(where the horizontal spacing emphasizes the runs of $y$ ). Note that $y_{w}$ depends on $w$ only through the locations of its Dyck words, not on which words they are, so for instance $y_{++--0+-0}=y_{+-+-0+-0}$.

Now let $y, z \in\{-,+\}^{n}$, and let $m$ be the number of runs of $y$. For $1 \leqslant j \leqslant$ $m-1$, let $\ell_{j}$ and $r_{j}$ be respectively the elements of $z$ immediately before and after the $j$ th sign change in $y$. For example, if

$$
\left(\begin{array}{l}
y \\
z
\end{array}\right)=\left(\begin{array}{cccccc}
++ & - & - & ++ & - & ++ \\
z_{1} z_{2} & z_{3} z_{4} z_{5} & z_{6} z_{7} & z_{8} & z_{9} z_{10}
\end{array}\right)
$$

then $\ell_{1}=z_{2}, r_{1}=z_{3}$, and $r_{3}=\ell_{4}=z_{8}$, and so forth. Let

$$
c(w, y, z):=\prod_{j=1}^{m-1} \begin{cases}\ell_{j} & w_{j}=+; \\ r_{j} & w_{j}=-; \\ 1 & w_{j}=0 .\end{cases}
$$

We are now ready to state the formula. For $x=\left(\begin{array}{l}y \\ z\end{array}\right) \in[4]^{n}$, where $y$ has $m$ runs, define

$$
Q(x)=Q\left(\begin{array}{l}
y \\
z
\end{array}\right):= \begin{cases}2^{n-m} \sum_{w \in \mathrm{DD}(m-1)}(-1)^{|w|} c(w, y, z) \alpha\left(y_{w}\right) & \text { if } x \text { is a } \\
0 & \text { proper coloring } \\
\text { otherwise }\end{cases}
$$

THEOREM 18. For $x \in[4]^{n}$ we have $B(x)=Q(x)$.

In consequence, the cylinder probabilities $P(x)$ for the 4-coloring $X$ of Theorem 1 can of course be expressed as $P(x)=Q(x) /\left[2^{n}(n+1) !\right]$. Theorem 18 will be proved by showing that $Q(x)$ satisfies the same recurrence as $B(x)$ (Lemma 9). It is now easy to deduce the claimed marginal distribution for the first binary digit. 
Proof of Theorem 4(iii). We claim that

$$
\sum_{z \in\{-,+\}^{n}} Q\left(\begin{array}{l}
y \\
z
\end{array}\right)=2^{n} \alpha(y), \quad y \in\{-,+\}^{n}
$$

then the result is immediate from Theorem 18.

To prove (19), sum (18) over $z$ and interchange the order of summation. The contribution from the trivial word $w=00 \cdots 0$ is

$$
\sum_{z: x \text { is proper }} 2^{n-m} \alpha(y)=2^{n} \alpha(y)
$$

since $z$ must alternate within each run of $y$, and thus there are $2^{m}$ choices. The contribution from every other $w$ vanishes. To see this, fix a nontrivial $w$, and consider the location of the first + in $w$. For any $z$, let $z^{\prime}$ be obtained from $z$ by changing the sign of every symbol in the run of $y$ that precedes that + . Then $c\left(w, y, z^{\prime}\right)=-c(w, y, z)$, so the terms corresponding to $z$ and $z^{\prime}$ cancel.

Theorem 18 implies a host of combinatorial identities; we briefly highlight some examples. Re-interpreting the result proved above in terms of buildings gives the following. For $y \in\{-,+\}^{n}$, define $S(y) \subset[4]^{n}$ to be the Cartesian product

$$
S(y):=\chi_{i=1}^{n} \begin{cases}\{1,2\} & y_{i}=-; \\ \{3,4\} & y_{i}=+.\end{cases}
$$

Then we have

$$
\sum_{x \in S(y)} B(x)=2^{n} \alpha(y), \quad y \in\{-,+\}^{n} .
$$

When $y=++\cdots+$ this is Corollary 12 with $q=2$, but it seems much less clear why the general case holds. Can it be given a bijective proof? Taking $y$ alternating of even length and combining with Theorem 4(iv) yields the curious identity

$$
\sum_{x \in(\{1,2\} \times\{3,4\})^{n}} B(x)=\frac{4^{n}}{n+1} \sum_{x \in(\{1,2\} \times\{3\})^{n}} B(x), \quad n \geqslant 1 .
$$

The $S_{4}$-symmetry of $B(x)$ implies in particular that

$$
Q\left(\begin{array}{l}
y \\
z
\end{array}\right)=Q\left(\begin{array}{l}
z \\
y
\end{array}\right), \quad y, z \in\{-,+\}^{n} .
$$

Again, it does not seem at all clear how to prove this directly from the definition (18). For instance, in the very simplest case where $z$ is a constant word and $y$ is 
alternating, it reduces to

$$
\sum_{\substack{m \geqslant 1, t_{1}, \ldots, t_{m} \geqslant 0: \\ \sum_{j}\left(2 t_{j}+1\right)=n}}\left[\prod_{j=1}^{m}\left(-C_{t_{j}}\right)\right] \alpha\left(2 t_{1}+1, \ldots, 2 t_{m}+1\right)=2^{n-1}, \quad n \geqslant 1,
$$

where $\alpha\left(k_{1}, \ldots, k_{m}\right)$ denotes $\alpha(y)$ for a word $y$ constructed so as to have successive run lengths $k_{1}, \ldots, k_{m}$, and $C_{t}:=\left(\begin{array}{c}2 t \\ t\end{array}\right) /(t+1)$ are the Catalan numbers. We have found a direct proof of this last identity, but even this involves a fairly intricate inclusion-exclusion argument for posets.

Another application of the formula (18) is that it gives rise to a computationally efficient method for computing the cylinder probabilities of the 4-coloring. Indeed, there is a recurrence based on (18) that allows $Q(x)(=B(x))$ to be computed in $O\left(n^{3}\right)$ operations for a word $x$ of length $n$, whereas a naïve application of (18) requires exponential time, as does computing $B(x)$ via Lemma 9. We state this recurrence at the end of this section.

Before giving the proof of Theorem 18 we briefly discuss how we arrived at the formula (18) (before knowing whether any $k$-dependent $q$-coloring existed). Suppose $X$ is a 1-dependent 4-coloring, and decompose it into two binary sequences $X=\left(\begin{array}{l}Y \\ Z\end{array}\right)$. Then $Y$ is a stationary 1-dependent binary process. The law of such a process is determined by the sequence $v_{n}=P\left(Y_{1}=\cdots=\right.$ $Y_{n}=+$ ), since all other cylinder probabilities can be computed from $v$ by inclusion-exclusion. Of course, the sequence $v$ must satisfy certain inequalities in order that these cylinder probabilities be nonnegative. Many choices for $v$ are possible. Examples are those for which $1,1, v_{1}, v_{2}, v_{3}, \ldots$ is a Pólya frequency sequence-see [29, Ch. 8].

Suppose for the purposes of the current discussion that $Y$ is any stationary 1-dependent binary process, and let $\alpha^{\prime}$ be defined by $\mathbb{P}\left[\left(Y_{1}, \ldots, Y_{n}\right)=y\right]=$ $\alpha^{\prime}(y) /(n+1)$ !. By considering the constraints imposed on the cylinder probabilities of $X$ by 1-dependence, one is led (after a certain amount of computation and some inspired guesses $)$ to the hypothesis that $\mathbb{P}\left[\left(X_{1}, \ldots\right.\right.$, $\left.\left.X_{n}\right)=x\right]=Q^{\prime}(x) /\left[(n+1) ! 2^{n}\right]$, where $Q^{\prime}$ is given in terms of $\alpha^{\prime}$ by the formula (18). It is not difficult to check that a $Q^{\prime}$ defined in this way satisfies the equalities required for consistency and 1-dependence of $X$, for any $\alpha^{\prime}$ arising from a stationary 1-dependent $Y$.

The only issue is nonnegativity of $Q^{\prime}(x)$. This does not hold for general $\alpha^{\prime}$ : for instance if $Y$ is independent and identically distributed with $\mathbb{P}\left(Y_{0}=+\right)=1 / 2$ then one can check that $Q^{\prime}(x)<0$ for $y=+-+-$ and $z=++++$. In fact it appears likely that $\alpha^{\prime}=\alpha$ is the only choice that works. However, it seems extremely difficult to prove nonnegativity of $Q$ directly from (18) in that case. The only way we know is to prove that $Q$ satisfies the same recurrence as $B$. 
We now turn to the proof of Theorem 18. A key ingredient is that $\alpha$ satisfies a recurrence similar to the one that we wish to check for $Q$. As before, let $\alpha\left(k_{1}, \ldots, k_{m}\right)$ denote $\alpha(y)$ where $y$ is a binary word with $m$ runs of successive lengths $k_{1}, \ldots, k_{m}$. If one $k_{i}$ is 0 the interpretation is that the two neighboring intervals coalesce, so that for example $\alpha\left(k_{1}, k_{2}, 0, k_{4}, k_{5}\right)=\alpha\left(k_{1}, k_{2}+k_{4}, k_{5}\right)$ and $\alpha\left(0, k_{2}, k_{3}, \ldots\right)=\alpha\left(k_{2}, k_{3}, \ldots\right)$.

PROPOSITION 19. For positive integers $k_{1}, \ldots, k_{m}$,

$$
\begin{aligned}
& \alpha\left(k_{1}, k_{2}, \ldots, k_{m}\right) \\
& \quad=\alpha\left(k_{1}-1, k_{2}, \ldots, k_{m}\right)+\alpha\left(k_{1}, k_{2}-1, \ldots, k_{m}\right)+\cdots+\alpha\left(k_{1}, \ldots, k_{m}-1\right) .
\end{aligned}
$$

This is a special case of the main result of [12], when applied to the poset that defines $\alpha$. We also give a simple direct proof.

Proof of Proposition 19. Suppose $\alpha\left(k_{1}, \ldots, k_{m}\right)=\alpha(y)$ where $y \in\{-,+\}^{n}$ is of length $n=\sum_{j} k_{j}$. Let $E$ be the set of permutations $\pi \in S_{n+1}$ that satisfy the inequalities in the definition of $\alpha(y)$, so $\alpha(y)=|E|$. For $1 \leqslant i \leqslant n+1$, let $E_{i}$ be the set of permutations $\pi \in E$ that have their maximum at $i$, that is $\pi_{i}=n+1$. For $1<i<n+1$ we further distinguish according to the order of the neighboring elements: let $E_{i}^{+}$be the set of $\pi \in E_{i}$ such that $\pi_{i-1}<\pi_{i+1}$, and define $E_{i}^{-}$similarly with the inequality reversed. Clearly,

$$
E=E_{1} \cup E_{n+1} \cup \bigcup_{1<i<n+1}\left(E_{i}^{+} \cup E_{i}^{-}\right),
$$

and the union is disjoint. However, $E_{i}$ is empty unless $\pi_{i}$ is already a local maximum in the sequence of inequalities defining $E$ (that is $\left(y_{i-1}, y_{i}\right)=$ $(+,-)$, where restrictions on ' $y_{0}$ ' and ' $y_{n+1}$ ' are ignored). In that case, we have

$$
\begin{aligned}
\left|E_{i}^{+}\right| & =\alpha\left(k_{1}, k_{2}, \ldots, \quad k_{j-1}, \quad k_{j}-1, \ldots, k_{m}\right) ; \\
\left|E_{i}^{-}\right| & =\alpha\left(k_{1}, k_{2}, \ldots, k_{j-1}-1, \quad k_{j}, \ldots, k_{m}\right),
\end{aligned}
$$

when $1<i<n+1$ and $\left(y_{i-1}, y_{i}\right)=(+,-)$ is the boundary between the $(j-1)$ st and $j$ th runs of $y$, and similar statements hold for $E_{1}$ and $E_{n+1}$. (Indeed, the maximum element $n+1$ in the permutation can be ignored, and the remaining elements $1, \ldots, n$ satisfy precisely the inequalities required for the appropriate 'reduced' $\alpha$.)

Proof of Theorem 18. Recall that $\widehat{x}_{i}$ denotes the word $x$ with the $i$ th symbol deleted. We claim that if $x \in[4]^{n}$ is a proper coloring,

$$
Q(x)=\sum_{i=1}^{n} Q\left(\widehat{x}_{i}\right) .
$$


Once this is proved, the result is immediate, since Lemma 9 states that $B$ satisfies the same recurrence, and $Q(\emptyset)=B(\emptyset)=1$ for the empty word $\emptyset$.

Let $x=\left(\begin{array}{l}y \\ z\end{array}\right)$ and let $y$ have $m$ runs. Since $z$ alternates within each run of $y$, we have $Q\left(\widehat{x}_{i}\right)=0$ whenever $i$ is an interior point of a run, because $\widehat{x}_{i}$ is not a proper coloring. So, we need to compute $Q\left(\widehat{x}_{i}\right)$ when $i$ is an endpoint of a run of $y$.

Suppose first that $i$ is an endpoint of a run of length at least 2, and suppose initially that it is not the first or last run. If, for example, $i$ is an endpoint of the $j$ th run of $y$, and that run is ---- , the relevant part of $x$ is

$$
x=\left(\begin{array}{l}
y \\
z
\end{array}\right)=\left(\begin{array}{cccc}
\cdots++ & + & - & - \\
\ell_{j-1} & r_{j-1} & \ell_{j} & r_{j}
\end{array}\right),
$$

and if $i$ is the left endpoint of that run, the corresponding $\widehat{x}_{i}$ is

$$
\widehat{x}_{i}=\left(\begin{array}{c}
\widehat{y}_{i} \\
\widehat{z}_{i}
\end{array}\right)=\left(\begin{array}{cccc}
\cdots++ & - & - & - \\
\ell_{j-1} & -r_{j-1} & \ell_{j} & r_{j}
\end{array}\right),
$$

while if $i$ is the right endpoint of that run,

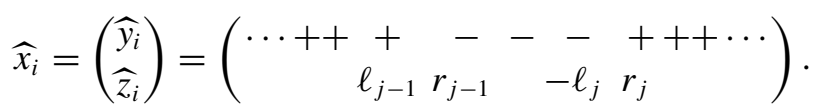

In passing from $x$ to $\widehat{x}_{i}$, the value of $m$ is unchanged, while the value of $n$ is decreased by 1 . In the first case above, the sign of $r_{j-1}$ is changed, while in the second case, the sign of $\ell_{j}$ is changed, and therefore

$$
c\left(w, \widehat{y}_{i}, \widehat{z}_{i}\right)=c(w, y, z)(-1)^{\mathbf{1}\left[w_{j-1}=-\right]}
$$

in the first case, and

$$
c\left(w, \widehat{y}_{i}, \widehat{z}_{i}\right)=c(w, y, z)(-1)^{1\left[w_{j}=+\right]}
$$

in the second. If we set $w_{0}=w_{m}=0$ then these also hold when the run is the first or the last. In both cases, $\widehat{y}_{i}$ is obtained from $y$ by shortening the corresponding run by 1 , and $\left(\widehat{y}_{i}\right)_{w}={\widehat{\left(y_{w}\right.}}_{i}$. Denote their common value by $\widehat{y}_{w, i}$. So, the contribution to the right side of (20) from (both endpoints of) this interval is

$$
2^{n-1-m} \sum_{w \in \mathrm{DD}(m-1)}(-1)^{|w|} c(w, y, z) \alpha\left(\widehat{y}_{w, i}\right)\left[(-1)^{1\left[w_{j-1}=-\right]}+(-1)^{1\left[w_{j}=+\right]}\right] .
$$

The last factor $(-1)^{1\left[w_{j-1}=-\right]}+(-1)^{1\left[w_{j}=+\right]}$ can be written as $2 I\left(w_{j-1}, w_{j}\right)$ where

$$
I(u, v):= \begin{cases}+1 & (u, v)=00 \text { or }+- \\ -1 & (u, v)=-+ \\ 0 & \text { otherwise }\end{cases}
$$


This follows simply by considering all possibilities for $\left(w_{j-1}, w_{j}\right)$, noting that $0-$ and +0 are impossible in a dispersed Dyck word. Therefore, (21) equals

$$
2^{n-m} \sum_{w \in \operatorname{DD}(m-1)}(-1)^{|w|} c(w, y, z) \alpha\left(\widehat{y}_{w, i}\right) I\left(w_{j-1}, w_{j}\right) .
$$

Now suppose $i$ is the sole element of a run of length 1. Again $n$ is decreased by 1 in passing from $x$ to $\widehat{x}_{i}$, but now $m$ decreases by 2 if $1<i<n$, or by 1 if $i \in\{1, n\}$. If $i=1$, each $w^{\prime} \in \mathrm{DD}(m-2)$ in the sum defining $Q\left(\widehat{x}_{1}\right)$ can be made into a $w \in \mathrm{DD}(m-1)$ by adding a 0 at the beginning, and this gives

$$
Q\left(\widehat{x}_{1}\right)=2^{n-m} \sum_{\substack{w \in \mathrm{DD}(m-1): \\ w_{1}=0}}(-1)^{|w|} c(w, y, z) \alpha\left(\widehat{y}_{w, 1}\right) .
$$

Similarly, for $i=n$, we add a 0 at the end:

$$
Q\left(\widehat{x}_{n}\right)=2^{n-m} \sum_{\substack{w \in \mathrm{DD}(m-1): \\ w_{m-1}=0}}(-1)^{|w|} c(w, y, z) \alpha\left(\widehat{y}_{w, n}\right) .
$$

If $1<i<n$, then (for example)

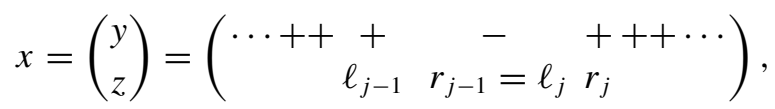

and

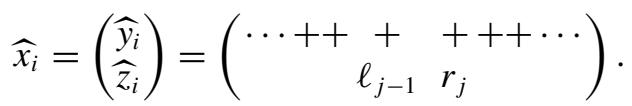

This is a proper coloring if and only if $\ell_{j-1} \neq r_{j}$. We will introduce a factor $\left(1-\ell_{j-1} r_{j}\right) / 2$ to account for this constraint. Let $w^{\prime} \in \mathrm{DD}(m-3)$ be a word in the sum corresponding to $Q\left(\widehat{x}_{i}\right)$. We can try to make $w^{\prime}$ into a word in $\mathrm{DD}(m-1)$ by inserting $00,+-$ or -+ before the $(j-1)$ st symbol of $w^{\prime}$; denote the resulting words $w_{00}, w_{+-}, w_{-+}$. Inserting +- introduces an additional factor $\ell_{j-1} r_{j}$ to $c$, and changes $\left|w^{\prime}\right|$ by 1 . Exactly one of $w_{00}, w_{-+}$is a dispersed Dyck word (inserting -+ succeeds precisely when there is a Dyck word that cannot be broken apart at the insertion point - note that for example +-+- can be broken in the middle, so here we would insert 00). Inserting 00 leaves $c$ and $\left|w^{\prime}\right|$ unchanged, while -+ multiplies $c$ by $r_{j-1} \ell_{j}=1$ and changes $\left|w^{\prime}\right|$ by 1 ; we introduce an extra sign change in this last case so that we can get the factor $\left(1-\ell_{j-1} r_{j}\right) / 2$. The conclusion is

$$
(-1)^{\left|w^{\prime}\right|} c\left(w^{\prime}, \widehat{y}_{i}, \widehat{z}_{i}\right) \frac{1-\ell_{j-1} r_{j}}{2}=\frac{1}{2} \sum_{\substack{w \in \mathrm{DD}(m-1) \cap \\\left\{w_{00}, w_{+-}, w_{-+}\right\}}}(-1)^{|w|} c(w, y, z)(-1)^{1\left[w=w_{-+}\right]} .
$$


Therefore,

$$
Q\left(\widehat{x}_{i}\right)=2^{n-m} \sum_{w \in \mathrm{DD}(m-1)}(-1)^{|w|} c(w, y, z) \alpha\left(\widehat{y}_{w, i}\right) I\left(w_{j-1}, w_{j}\right),
$$

where $I\left(w_{j-1}, w_{j}\right)$ is precisely the same quantity as defined for the earlier case, and where the factor $1 / 2$ has canceled the extra 2 in $2^{(n-1)-(m-2)}$. Finally, note that if we again set $w_{0}=w_{m}=0$ then (23) is valid in the cases $i=1, n$ also.

For each $1 \leqslant j \leqslant m$, write $\widetilde{y}_{w, j}=\widehat{y}_{w, i}$, where $i=i(j)$ is in the $j$ th run of $y$. This is the same for all runs $j$ that coalesce into a single run when we form $y_{w}$. Summing over all runs of $y$, we see that the right side of (20) can be written as

$$
2^{n-m} \sum_{w \in \mathrm{DD}(m-1)}(-1)^{|w|} c(w, y, z) \sum_{j=1}^{m} \alpha\left(\tilde{y}_{w, j}\right) I\left(w_{j-1}, w_{j}\right) .
$$

Each Dyck word in $w$ corresponds to a run of $y_{w}$, as does each 00 (where again we take $w_{0}=w_{m}=0$ ). Every Dyck word contains exactly one more +- than -+ . Therefore, the sum of $I\left(w_{j-1}, w_{j}\right)$ over those $j$ that correspond to a given run of $y_{w}$ is 1 . By Proposition 19, the right side of (20) agrees with $Q(x)$.

Finally, we state the promised alternative recurrence for $Q$ that allows for efficient computation. We have for all proper colorings $x \in[4]^{n}$,

$$
Q(x)=\sum_{r=1}^{n+1} Q_{r}^{0}(x)
$$

where the quantity $Q_{r}^{k}(x)=Q_{r}^{k}\left(\begin{array}{l}y \\ z\end{array}\right)$ is defined for integers $k \geqslant 0$ and $1 \leqslant r \leqslant n+1$ by

$$
Q_{r}^{k}(x)=\mathbf{1}\left[k=0 \text { and } y_{1}=(-1)^{r+1}\right], \quad n=1,
$$

and for $n \geqslant 2$,

$$
Q_{r}^{k}(x)=\sum_{s \in S} \begin{cases}2 Q_{s}^{k}\left(\widehat{x}_{1}\right) & y_{1}=y_{2} \\ Q_{s}^{k}\left(\widehat{x}_{1}\right)-z_{1} Q_{s}^{k+1}\left(\widehat{x}_{1}\right) & y_{1} \neq y_{2} \text { and } k=0 \\ z_{2} Q_{s}^{k-1}\left(\widehat{x}_{1}\right)-z_{1} Q_{s}^{k+1}\left(\widehat{x}_{1}\right) & y_{1} \neq y_{2} \text { and } k>0,\end{cases}
$$

where

$$
S:= \begin{cases}\{r, \ldots, n\} & y_{1}=(-1)^{k} \\ \{1, \ldots, r-1\} & y_{1}=(-1)^{k+1} .\end{cases}
$$

We omit the proof of this, which is a straightforward check given the following explanation. The quantity $Q_{r}^{k}(x)$ represents an extended version of $Q(x)$ in 
which we sum over 'partial dispersed Dyck words' $w$ that can be made into a dispersed Dyck word by appending exactly $k+$ 's at the beginning, and where in addition each $\alpha\left(y_{w}\right)$ is modified by restricting to permutations $\pi \in S_{n+1}$ satisfying $\pi_{1}=r$.

\section{Higher dimensions and shifts of finite type}

In this section, we prove Corollaries 5 and 6 . Let $\|\cdot\|=\|\cdot\|_{1}$ be the 1-norm on $\mathbb{Z}^{d}$. The distance between two sets $A, B \subseteq \mathbb{Z}^{d}$ is $\inf \{\|u-v\|: u \in A, v \in$ $B\}$. We first observe that the definition of $k$-dependence for graphs given in the introduction is consistent with the earlier definition for $\mathbb{Z}$. Indeed, suppose $X$ is $k$-dependent according to the earlier definition. Then if $\left(I_{j}\right)_{j \in J}$ is any collection of intervals of $\mathbb{Z}$ no two of which are within distance $k$ then the restrictions $\left(\left.X\right|_{I_{j}}\right)_{j \in J}$ form an independent family; this follows by inductively adding one interval at a time. Now if $A, B \subseteq \mathbb{Z}$ are at distance greater than $k$ then $\left.X\right|_{A}$ and $\left.X\right|_{B}$ are independent, since $A$ and $B$ can each be partitioned into subsets that are contained in such a collection of intervals.

We need the following extension of Theorem 1. Write $u \stackrel{m}{\sim} v$ if $0<\|u-v\|$ $\leqslant m$. A process $\left(X_{v}\right)_{v \in \mathbb{Z}^{d}}$ is a range- $\boldsymbol{m} \boldsymbol{q}$-coloring if each $X_{v}$ takes values in $[q]$, and almost surely $X_{u} \neq X_{v}$ whenever $u \stackrel{m}{\sim} v$. (A range- 1 coloring is simply a coloring.)

COROLlARY 20. Let $d \geqslant 1$ and $m \geqslant 1$. There exists a stationary $m$-dependent range-m $q$-coloring of $\mathbb{Z}^{d}$, where $q \leqslant \exp \left(\mathrm{cm}^{d}\right)$ for an absolute constant $c$.

Proof. A line is a subset of $\mathbb{Z}^{d}$ of the form $L=\{a+i h: i \in \mathbb{Z}\}$, where $a, h \in \mathbb{Z}^{d}$ and $h \neq 0$. We call $h$ the direction of $L$. We will place independent copies of the 1-dependent 4-coloring along each line in a suitable family, and combine them to form the desired process.

More precisely, let $H$ be a set comprising exactly one of $h$ and $-h$ for every $h \in \mathbb{Z}^{d}$ with $0 \stackrel{m}{\sim} h$. (For instance, in the case $m=1$ we can take $H$ to be the set of $d$ standard basis vectors.) For each line $L$ of $\mathbb{Z}^{d}$ with direction in $H$, take a copy $X^{L}$ of the 1-dependent 4-coloring of Theorem 1, with the copies being independent for different lines. Assign the color $X_{j}^{L}$ to the point $a+j h$, where $L=\{a+i h: i \in \mathbb{Z}\}$ (and $a \in L$ is chosen arbitrarily, but is deterministic and fixed for the particular line). Let $Y_{v}^{h} \in$ [4] denote the color thus assigned to $v \in \mathbb{Z}^{d}$ by the unique line of direction $h$ passing through $v$. Finally define $Z_{v}$ to be the vector $\left(Y_{v}^{h}: h \in H\right) \in[4]^{H}$. The desired process is $Z=\left(Z_{v}\right)_{v \in \mathbb{Z}^{d}}$.

Clearly $Z$ is stationary, and its elements take $4^{|H|}$ values. It is a range- $m$ coloring since for any $u, v$ with $u \stackrel{m}{\sim} v$ there is a line on which $u, v$ are 
consecutive points, so $Z_{u}$ and $Z_{v}$ differ in the coordinate corresponding to its direction. (Two points on a line of direction $h$ are said to be consecutive on the line if they differ by $\pm h$.) To check $m$-dependence, note that if $A, B \subseteq \mathbb{Z}^{d}$ are at distance greater than $m$ from each other then every line with direction in $H$ that intersects both $A$ and $B$ does so in two nonconsecutive sets. Thus $\left.Z\right|_{A}$ and $\left.Z\right|_{B}$ are functions of independent collections of random variables.

Proof of Corollary 5(i). This is Corollary 20 with $m=1$. (The number of colors is $q=4^{d}$.)

To state the relevant results from [22] we need to generalize block factors to $d$ dimensions. Denote the ball $B(r):=\left\{v \in \mathbb{Z}^{d}:\|v\| \leqslant r\right\}$. A block-factor map is a map $F: \mathbb{R}^{\mathbb{Z}^{d}} \rightarrow \mathbb{R}^{\mathbb{Z}^{d}}$ characterized by an integer $r$ called the radius and a measurable function $f: \mathbb{R}^{B(r)} \rightarrow \mathbb{R}$ via

$$
(F(x))_{v}=f\left(\left.\left(\theta^{-v} x\right)\right|_{B(r)}\right), \quad x \in \mathbb{R}^{\mathbb{Z}^{d}}, v \in \mathbb{Z}^{d},
$$

where $\theta^{-v}$ denotes translation by $-v\left(\right.$ so $\left.\left(\theta^{-v} x\right)_{u}=x_{v+u}\right)$. (On $\mathbb{Z}$, a radius$r$ block-factor map of an independent and identically distributed process is a $(2 r+1)$-block-factor, in our earlier terminology.)

LEMMA 21. Let $X$ be a stationary $k$-dependent process on $\mathbb{Z}^{d}$ and let $F$ be a radius-r block-factor map. Then $F(X)$ is stationary and $(2 r+k)$-dependent.

Proof. This follows easily from the definitions.

THEOREM 22 (Holroyd et al. [22]).

(i) Let $d \geqslant 1$. There exists $m$ such that for any $q$ there exists a block-factor map $F$ with the following property. If $X$ is a range-m $q$-coloring of $\mathbb{Z}^{d}$ then $F(X)$ is a (range-1) 4-coloring of $\mathbb{Z}^{d}$.

(ii) Let $S$ be a nonlattice shift of finite type on $\mathbb{Z}$. There exists $m$ such that for any $q$ there exists a block-factor map $F$ with the following property. If $X$ is a range-m $q$-coloring of $\mathbb{Z}$ then $F(X)$ belongs to $S$ almost surely.

The somewhat awkward series of quantifiers above reflects the need to encapsulate the relevant results from [22] cleanly without going into details of their proofs.

Proof of Corollary 5(ii) and Corollary 6. The results are immediate from Corollary 20, Lemma 21, and Theorem 22. 
We make a few remarks about the scope of Corollaries 5 and 6. While the colorings of Corollary 5 are stationary (meaning invariant under translations), they are not invariant in law under all isometries of $\mathbb{Z}^{d}$, because the proof imposed an ordering on the set of line directions, which is not invariant under permuting the coordinates. We do not know how to construct an isometryinvariant finitely dependent coloring of $\mathbb{Z}^{d}$ for $d \geqslant 2$. Similar remarks apply to trees, as pointed out by Russell Lyons (personal communication). Treating a regular tree as the Cayley graph of a free group, we obtain a 1-dependent coloring that is invariant under the action of the group itself (which is vertex-transitive), by the same approach as in the proof of Corollary 5. However, we do not know how to construct a fully automorphism-invariant finitely dependent coloring.

As remarked in the introduction, another result of [22] implies that there is no stationary $k$-dependent 3 -coloring of $\mathbb{Z}^{d}$ for any $k$ and $d \geqslant 2$. In fact, there is no stationary 3 -coloring of $\mathbb{Z}^{2}$ whose correlations decay faster than a certain polynomial rate.

It is straightforward to check that if $S$ is a lattice shift of finite type on $\mathbb{Z}$ then there is no stationary finitely dependent process that belongs almost surely to $S$. In fact, there is no stationary mixing process that belongs to $S$; again, details appear in [22].

\section{One-dependent hard-core processes}

In this section, we prove Proposition 7. We also discuss properties of 1-dependent hard-core processes, which are interesting in their own right. Let $G=(V, E)$ be a simple, countable, undirected graph with all degrees finite. Recall that a hard-core process $J=\left(J_{v}\right)_{v \in V}$ is a $\{0,1\}$-valued process with no adjacent 1's, and that $p_{\mathrm{h}}(G)$ is defined to be the supremum of $p$ for which there exists a 1-dependent hard-core process with all its one-vertex marginals $\mathbb{P}\left(J_{v}=1\right)$ equal to $p$.

In Lemmas 23 and 24 below we record some simple but interesting observations about $p_{\mathrm{h}}$. Closely related ideas appear in work of Scott and Sokal $[42,43]$, where a rich web of interconnections involving mathematical physics and probabilistic combinatorics is explored. The arguments we use in the proofs of Lemmas 23 and 24 are largely present in those articles, at least implicitly. However, our particular viewpoint (focussing on 1-dependent hardcore processes, especially on infinite graphs) is apparently novel, as is our application to coloring. As another application of our approach, we give an alternative proof of a result of Shearer [44] at the end of this section. 
LeMmA 23. Let $G$ be a graph. For each $p \leqslant p_{\mathrm{h}}$ there exists a unique 1-dependent hard-core process with all one-vertex marginals equal to $p$. This process is invariant in law under all automorphisms of $G$.

Proof. We first observe a general monotonicity statement: if a 1-dependent hardcore process $J$ with one-vertex marginals $\mathbb{P}\left(J_{v}=1\right)=p_{v}$ exists, and if $p_{v}^{\prime} \leqslant p_{v}$ for all $v \in V$, then such a process exists with marginals $\left(p_{v}^{\prime}\right)$. This follows by thinning: let $\left(\epsilon_{v}\right)_{v \in V}$ be $\{0,1\}$-valued, independent of each other and of $J$, with $\mathbb{P}\left(\epsilon_{v}=1\right)=p_{v}^{\prime} / p_{v}$; then take $J_{v}^{\prime}=\epsilon_{v} J_{v}$.

The above shows that a 1-dependent hard-core process exists for all $p<p_{\mathrm{h}}$. To extend this to $p=p_{\mathrm{h}}$, take a sequence $p_{n} \nearrow p_{\mathrm{h}}$ and a process for each $p_{n}$, and consider a subsequential weak limit in distribution $J$ (which exists, by compactness). Since probabilities of all cylinder events converge, $J$ has all marginals equal to $p_{\mathrm{h}}$, and is a 1-dependent hard-core process.

Uniqueness and automorphism invariance follow from the more general fact that the law of a 1-dependent hard-core process is determined by its onevertex marginals $p_{v}=\mathbb{P}\left(J_{v}=1\right)$. Indeed, the law of a binary process $J$ is determined by the probabilities $\mathbb{P}(J \equiv 1$ on $A)$ for finite $A \subseteq V$, since all other cylinder probabilities can be computed from them by inclusion-exclusion. But this probability equals 0 if $A$ contains two neighbors, and otherwise it is $\prod_{v \in A} p_{v}$.

We next give a simple characterization of $p_{h}$ in terms of finite sets. (Such characterizations can be powerful tools in understanding phase transitions-see [11] for a notable recent example.) For a finite set of vertexes $A \subseteq V$ and $\lambda \in \mathbb{R}$, define

$$
Z_{A}(\lambda):=\sum_{B \in \mathcal{I}(A)} \lambda^{|B|}
$$

where $\mathcal{I}$ is the set of all independent subsets of $A$ (or hard-core configurations), that is subsets of $A$ that do not contain any two neighbors in $G$. This is the partition function of the standard hard-core model of statistical physics; it is also known as the independence polynomial of the induced subgraph of $A$. See for example [31, 43].

LEMMA 24. Let $G=(V, E)$ be a graph and let $p \in[0,1]$. We have $p \leqslant p_{h}$ if and only if $Z_{A}(-p) \geqslant 0$ for every finite $A \subseteq V$. If $G$ is infinite and connected then this is also equivalent to the statement that the strict inequality $Z_{A}(-p)>0$ holds for every finite $A \subset V$. 
Proof. Suppose that $p \leqslant p_{\mathrm{h}}$, so a 1-dependent hard-core process $J$ with marginals $p$ exists. Then by inclusion-exclusion,

$$
\mathbb{P}(J \equiv 0 \text { on } A)=\sum_{B \subseteq A}(-1)^{|B|} \mathbb{P}(J \equiv 1 \text { on } B)=Z_{A}(-p),
$$

so the last quantity is nonnegative.

Moreover, all other cylinder probabilities can be expressed in terms of those above. Let $B, C$ be disjoint finite sets of vertexes with $B \in \mathcal{I}(V)$, and let $C^{\prime}$ be the set of vertexes of $C$ that have no neighbor in $B$. Then

$$
\begin{aligned}
\mathbb{P}(J \equiv 1 \text { on } B, J \equiv 0 \text { on } C) & =\mathbb{P}\left(J \equiv 1 \text { on } B, J \equiv 0 \text { on } C^{\prime}\right) \\
& =p^{|B|} Z_{C^{\prime}}(-p) .
\end{aligned}
$$

Thus, given $Z_{A}(-p) \geqslant 0$ for all $A$, we can compute nonnegative expressions for all cylinder probabilities, and it is easy to check that they are consistent and give rise to a 1-dependent hard-core process with marginals $p$. Thus $p \leqslant p_{\mathrm{h}}$.

Here is a useful recurrence. Suppose $A \subseteq V$ is finite, let $u \in A$, and define $A^{\prime}:=A \backslash\{u\}$ and $A^{\prime \prime}:=A^{\prime} \backslash N(u)$, where $N(u)$ denotes the set of neighbors of $u$. Then by an argument similar to the above,

$$
Z_{A}(-p)=Z_{A^{\prime}}(-p)-p Z_{A^{\prime \prime}}(-p) .
$$

(Indeed, it is a standard and straightforward fact that this identity holds for any parameter $\lambda$, regardless of the existence of the process $J$; see for example $[31,43])$.

To prove the final claimed equivalence, suppose that $G$ is infinite and connected. Let $0<p \leqslant p_{\mathrm{h}}$. (If $p_{\mathrm{h}}=0$ then the claim is trivial.) Suppose that $Z_{A}(-p)=0$ for some finite $A \subset V$, and let $A$ be minimal with this property. There exists a vertex $u \notin A$ that is adjacent to $A$. Let $B=A \cup\{u\}, B^{\prime}=A$, and $B^{\prime \prime}=A \backslash N(u)$. Then applying (25) to $B, B^{\prime}, B^{\prime \prime}$ gives that $Z_{B}(-p)$ is negative, a contradiction.

For an infinite connected $G$, our critical point $p_{\mathrm{h}}$ coincides with the critical point $\lambda_{\mathrm{c}}$ defined in [43] (in (5.3) and the immediately following remark) in terms of the complex zeros of $Z$. This follows immediately from Lemma 24 above together with [43, Theorem 2.2(b,c) and (3.1)].

The following bounds on $p_{\mathrm{h}}$ are known. For any infinite connected graph $G$ of maximum degree $\Delta$,

$$
\frac{(\Delta-1)^{\Delta-1}}{\Delta^{\Delta}} \leqslant p_{\mathrm{h}}(G) \leqslant \frac{1}{4}, \quad \Delta \geqslant 2 .
$$


For the infinite $\Delta$-regular tree $T_{\Delta}$, the lower bound is sharp:

$$
p_{\mathrm{h}}\left(T_{\Delta}\right)=\frac{(\Delta-1)^{\Delta-1}}{\Delta^{\Delta}}, \quad \Delta \geqslant 2 .
$$

For the hypercubic lattice $\mathbb{Z}^{d}$,

$$
\frac{(2 d-1)^{2 d-1}}{(2 d)^{2 d}} \leqslant p_{\mathrm{h}}\left(\mathbb{Z}^{d}\right) \leqslant \frac{d^{d}}{(d+1)^{d+1}}, \quad d \geqslant 1 .
$$

Proofs of (26), (28) appear in [42, Sections 5.2, 8.4]; the lower bound in (26) amounts to the Lovász local lemma. The equality (27) is proved in [44], and an exposition of the proof also appears in $[42,43]$. Note that $p_{\mathrm{h}}(\mathbb{Z})=1 / 4$. This is a special case of all of (26), (27), (28), and also follows from the proof of Corollary 17. Using rigorous computer-assisted methods, we supply the following improvement on (28) in dimensions 2 and 3.

LEMMA 25. We have the strict inequalities

$$
p_{\mathrm{h}}\left(\mathbb{Z}^{2}\right)<\frac{1}{8} ; \quad p_{\mathrm{h}}\left(\mathbb{Z}^{3}\right)<\frac{1}{11} .
$$

Proof. The recursion (25) gives $Z_{A}(-p)$ in terms of $Z_{B}(-p)$ for smaller sets $B \subset A$. We use this to compute $Z_{A}(-p)$ numerically for rectangular boxes of the form $A=[a] \times[b] \subset \mathbb{Z}^{2}$ and $A=[a] \times[b] \times[c] \subset \mathbb{Z}^{3}$. After some experimentation to find appropriate box sizes, we obtained

$$
Z_{[13] \times[10]}(-1 / 8)<0 ; \quad Z_{[12] \times[4] \times[4]}(-1 / 11)<0,
$$

giving the claimed bounds.

One must choose which vertex $u$ to remove from a set $A$ when applying (25). We always chose the lexicographically largest $u \in A$, as this tends to limit the number of smaller sets that need to be considered. The method turns out to be numerically unstable, so that floating-point arithmetic cannot be used. Instead we used exact arbitrary-precision rational arithmetic. The quantity $Z_{[12] \times[4] \times[4]}(-1 / 11)$ is a fraction with 100 digits in the denominator, and required the computation of $Z_{B}(-1 / 11)$ for 89077 sets $B \subseteq[12] \times$ [4] $\times$ [4]. (We provide the computer code in an appendix.)

Proof of Proposition 7. As remarked in the introduction, the existence of a 1-dependent $q$-coloring $X$ with the variables $\left(X_{v}\right)_{v \in V}$ identically distributed implies that $q \geqslant 1 / p_{\mathrm{h}}$. Indeed, let $a \in[q]$ be a color with the largest marginal probability $p_{a}(\geqslant 1 / q)$; then $J_{v}:=\mathbf{1}\left[X_{v}=a\right]$ defines a 1-dependent hard-core process, so $p_{a} \leqslant p_{\mathrm{h}}$. Now use the upper bounds in (27), (28) and Lemma 25. 
The (nonrigorous) estimate $p_{\mathrm{h}}\left(\mathbb{Z}^{2}\right)=0.11933888188(1)$ was computed in [46]. That this is greater than $1 / 9$ indicates that a 9-coloring of $\mathbb{Z}^{2}$ cannot be ruled out by the methods of this section.

Finally, we present an application of our approach in the context of [43]. Motivated by the case of $\mathbb{Z}$ in Theorem 4(ii), we give a very simple explicit construction of the critical 1-dependent hard-core process $J$ on the $\Delta$-regular tree $T_{\Delta}$, thus providing an alternative proof of the upper bound on $p_{\mathrm{h}}\left(T_{\Delta}\right)$ in (27). (The original proof in [44] used analytic methods.) Fix an end of the tree. Assign the vertexes independent and identically distributed $\{0,1\}$-valued labels that are 1 with probability $1 / \Delta$. Then let $J_{v}$ equal 1 if and only if $v$ has label 1 and all its children have label 0 . (The children of a vertex are the $\Delta-1$ neighbors that do not lie on the unique path to the nominated end.) Then $\mathbb{P}\left(J_{v}=1\right)=(\Delta-1)^{\Delta-1} / \Delta^{\Delta}$ as required. It is interesting that the construction is invariant only under automorphisms that fix the given end, while the process itself is fully automorphism-invariant, by Lemma 23. Can the critical process on $T_{\Delta}$ be expressed as a fully automorphism-equivariant block factor of an independent and identically distributed process?

\section{Open problems}

(i) Is the stationary 1-dependent 4-coloring of $\mathbb{Z}$ unique? We conjecture that the answer is yes. Is the stationary 2-dependent 3-coloring unique?

(ii) Is there a finitely dependent coloring $\left(X_{i}\right)_{i \in \mathbb{Z}}$ such that $X_{i}=f\left(M_{i}\right)$ for a stationary countable-state Markov chain $M$ ? (A finite state space is impossible, while an uncountable one places no restriction on the process.) Can our two examples be expressed in this way?

(iii) What is the largest possible one-vertex marginal of a stationary $k$ dependent hard-core process on $\mathbb{Z}$ for $k \geqslant 2$ ? Is it $1 / 3$ when $k=2$ ? Is the critical process unique?

(iv) Can one of our two colorings of $\mathbb{Z}$ be expressed as a block factor of the other? As a finitary factor?

(v) Is there a stationary finitely dependent coloring of $\mathbb{Z}$ that can be expressed as a finitary factor of an independent and identically distributed process with finite mean coding radius? (In [20], the 4-coloring is expressed as a finitary factor with infinite mean coding radius.)

(vi) What is the minimum number of colors $q$ needed for a stationary 1dependent $q$-coloring of $\mathbb{Z}^{d}$, for each $d \geqslant 2$ ? (For $\mathbb{Z}^{2}$, the answer is between 9 and 16.) 
(vii) Does there exist a finitely dependent coloring of $\mathbb{Z}^{d}$ for $d \geqslant 2$ that it is invariant in law under all isometries of $\mathbb{Z}^{d}$ ? Does there exist a finitely dependent coloring of a regular tree that is invariant under all automorphisms, or all automorphisms that fix a given end?

(viii) On which transitive graphs is the existence of a 1-dependent hard-core process with all one-vertex marginals equal to $1 / q$ sufficient for the existence of an automorphism-invariant 1-dependent $q$-coloring? (It is necessary on any graph, and sufficient on $\mathbb{Z}$.)

\section{Acknowledgments}

We thank Itai Benjamini, David Brydges, Ronen Eldan, Jeong-Han Kim, Russell Lyons, Ben Morris, Fedja Nazarov, Robin Pemantle, Benjamin Weiss, Peter Winkler and Fuxi Zhang for valuable discussions. We thank the referees for helpful suggestions.

\section{Appendix: Computer code}

Below we give Python code used in the proof of Lemma 25. It computes the following values of the independence polynomial for rectangular grids. (The first is included as a check.)

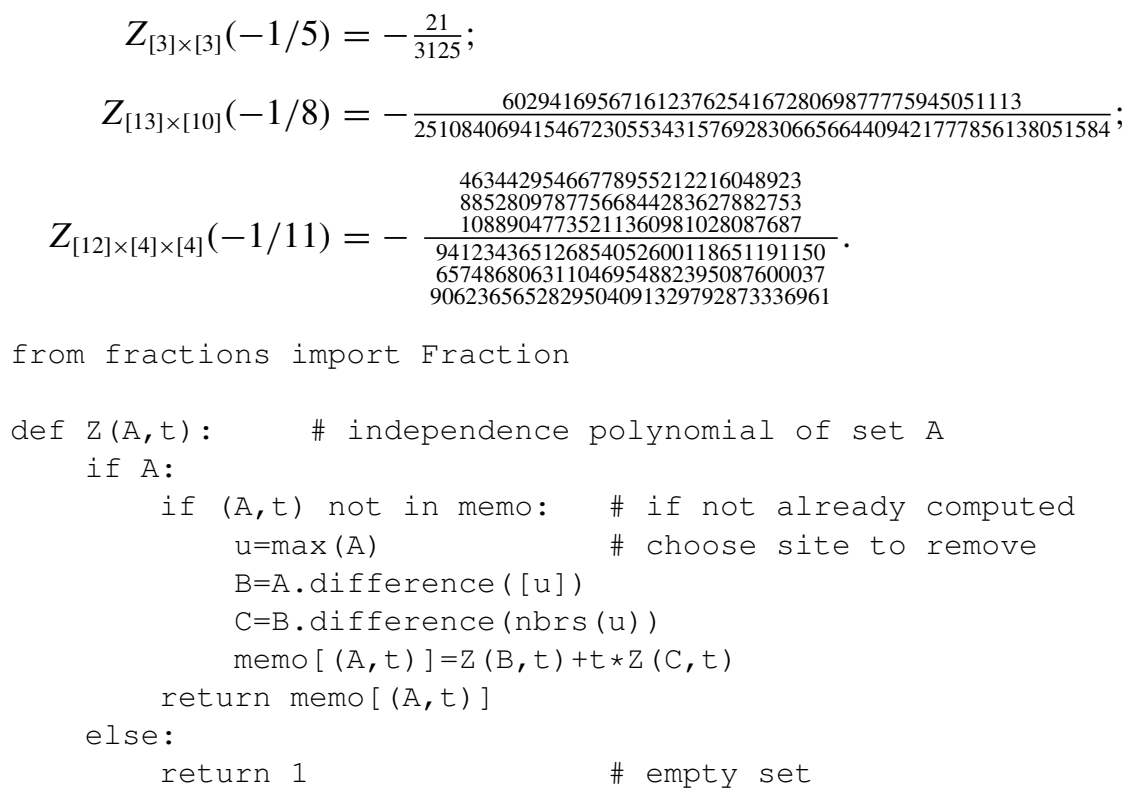




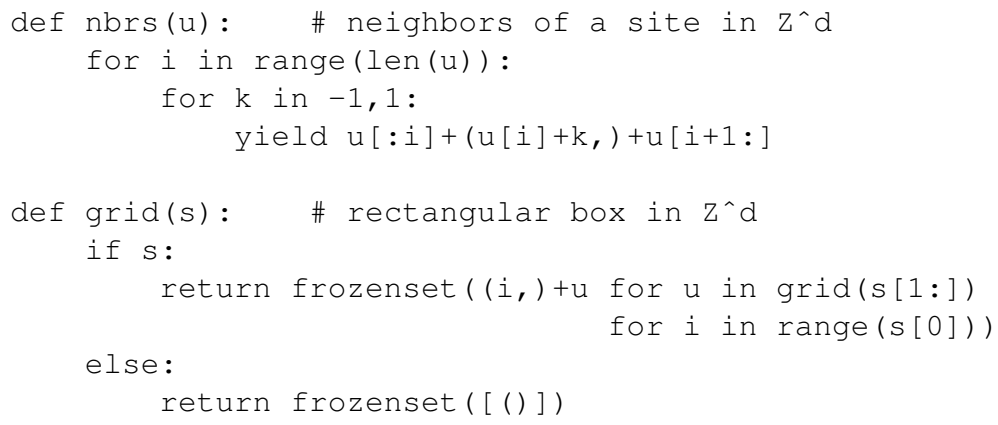

\section{References}

[1] J. Aaronson, D. Gilat and M. Keane, 'On the structure of 1-dependent Markov chains', J. Theoret. Probab. 5(3) (1992), 545-561.

[2] J. Aaronson, D. Gilat, M. Keane and V. de Valk, 'An algebraic construction of a class of one-dependent processes', Ann. Probab. 17(1) (1989), 128-143.

[3] N. Alon and O. N. Feldheim, 'A note on general sliding window processes', Electron. Commun. Probab. 19(66) (2014), 1-7.

[4] N. Alon and J. H. Spencer, The Probabilistic Method, 3rd edn, Wiley-Interscience Series in Discrete Mathematics and Optimization, (John Wiley \& Sons, Inc., Hoboken, NJ, 2008), With an appendix on the life and work of Paul Erdős.

[5] S. Billey, K. Burdzy and B. E. Sagan, 'Permutations with given peak set', J. Integer Seq. 16(6) (2013), Article 13.6.1, 18.

[6] A. Borodin, P. Diaconis and J. Fulman, 'On adding a list of numbers (and other onedependent determinantal processes)', Bull. Amer. Math. Soc. (N.S.) 47(4) (2010), 639-670.

[7] E. I. Broman, 'One-dependent trigonometric determinantal processes are two-block-factors', Ann. Probab. 33(2) (2005), 601-609.

[8] R. M. Burton, M. Goulet and R. Meester, 'On 1-dependent processes and $k$-block factors', Ann. Probab. 21(4) (1993), 2157-2168.

[9] V. de Valk, 'A problem on 0-1 matrices', Compositio Math. 71(2) (1989), 139-179.

[10] V. de Valk, 'Hilbert space representations of $m$-dependent processes', Ann. Probab. 21(3) (1993), 1550-1570.

[11] H. Duminil-Copin and V. Tassion, 'A new proof of the sharpness of the phase transition for Bernoulli percolation and the Ising model', Comm. Math. Phys. 343(2) (2016), 725-745.

[12] P. Edelman, T. Hibi and R. P. Stanley, 'A recurrence for linear extensions', Order 6(1) (1989), $15-18$. 
[13] P. Erdős and L. Lovász, 'Problems and results on 3-chromatic hypergraphs and some related questions', in Infinite and Finite Sets, Vol. II, Colloq. Math. Soc. János Bolyai, 10 (NorthHolland, Amsterdam, 1975), 609-627.

[14] P. Flajolet and R. Sedgewick, Analytic Combinatorics (Cambridge University Press, Cambridge, 2009).

[15] A. Gandolfi, M. Keane and V. de Valk, 'Extremal two-correlations of two-valued stationary one-dependent processes', Probab. Theory Related Fields 80(3) (1989), 475-480.

[16] F. Götze and C. Hipp, 'Asymptotic expansions for potential functions of i.i.d. random fields', Probab. Theory Related Fields 82(3) (1989), 349-370.

[17] M. G. Haiman, 'Valeurs extrémales de suites stationnaires de variables aléatoires $m$ dépendantes', Ann. Inst. H. Poincaré Sect. B (N.S.) 17(3) (1981), 309-330.

[18] L. Heinrich, 'Asymptotic expansions in the central limit theorem for a special class of $m$ dependent random fields. II. Lattice case', Math. Nachr. 145 (1990), 309-327.

[19] W. Hoeffding and H. Robbins, 'The central limit theorem for dependent random variables', Duke Math. J. 15 (1948), 773-780.

[20] A. E. Holroyd, One-dependent coloring by finitary factors. Ann. Inst. Henri Poincaré, arXiv:1411.1463.

[21] A. E. Holroyd and T. M. Liggett, 'Symmetric 1-dependent colorings of the integers', Electron. Commun. Probab. 20(31) (2015), 1-8.

[22] A. E. Holroyd, O. Schramm and D. B. Wilson, Finitary coloring. Ann. Probab., arXiv:1412.2725.

[23] I. A. Ibragimov and Yu. V. Linnik, Nezavisimye stalionarno svyazannye velichiny, Izdat, (Nauka, Moscow, 1965).

[24] I. A. Ibragimov and Yu. V. Linnik, Independent and Stationary Sequences of Random Variables (Wolters-Noordhoff Publishing, Groningen, 1971), With a supplementary chapter by I. A. Ibragimov and V. V. Petrov, Translation from the Russian edited by J. F. C. Kingman.

[25] S. Janson, 'Renewal theory for M-dependent variables', Ann. Probab. 11(3) (1983), $558-568$.

[26] S. Janson, 'Runs in m-dependent sequences', Ann. Probab. 12(3) (1984), 805-818.

[27] S. Janson, 'On degenerate sums of $m$-dependent variables', J. Appl. Probab. 52(4) (2015), 1146-1155.

[28] O. Kallenberg, Foundations of Modern Probability, 2nd edn, Probability and its Applications (Springer, New York, 2002).

[29] S. Karlin, Total Positivity, Vol. I (Stanford University Press, Stanford, CA, 1968).

[30] P. D. Lax, Functional Analysis, Pure and Applied Mathematics (Wiley-Interscience, New York, 2002).

[31] V. E. Levit and E. Mandrescu, 'The independence polynomial of a graph-a survey', in Proceedings of the 1st International Conference on Algebraic Informatics (Aristotle Univ. Thessaloniki, Thessaloniki, 2005), 233-254.

[32] T. M. Liggett, R. H. Schonmann and A. M. Stacey, 'Domination by product measures', Ann. Probab. 25(1) (1997), 71-95.

[33] N. Linial, 'Distributive graph algorithms - global solutions from local data', in 28th Annual Symposium on Foundations of Computer Science (IEEE, 1987), 331-335.

[34] C. Mallows and L. Shepp, 'The necklace process', J. Appl. Probab. 45(1) (2008), 271-278.

[35] F. Matúš, 'On two-block-factor sequences and one-dependence', Proc. Amer. Math. Soc. 124(4) (1996), 1237-1242.

[36] F. Matúš, 'Combining $m$-dependence with Markovness', Ann. Inst. Henri Poincaré Probab. Stat. 34(4) (1998), 407-423. 
[37] T. Nakata, 'Necklace processes via Pólya urns', J. Appl. Probab. 46(1) (2009), 284-295.

[38] M. Naor, 'A lower bound on probabilistic algorithms for distributive ring coloring', SIAM J. Discrete Math. 4(3) (1991), 409-412.

[39] I. Niven, 'A combinatorial problem of finite sequences', Nieuw Arch. Wiskd. (3) 16 (1968), $116-123$.

[40] G. L. O'Brien, 'Scaling transformations for $\{0,1\}$-valued sequences', Z. Wahrsch. Verw. Gebiete 53(1) (1980), 35-49.

[41] L. Rüschendorf and V. de Valk, 'On regression representations of stochastic processes', Stochastic Process. Appl. 46(2) (1993), 183-198.

[42] A. D. Scott and A. D. Sokal, 'The repulsive lattice gas, the independent-set polynomial, and the Lovász local lemma', J. Stat. Phys. 118(5-6) (2005), 1151-1261.

[43] A. D. Scott and A. D. Sokal, 'On dependency graphs and the lattice gas', Combin. Probab. Comput. 15(1-2) (2006), 253-279.

[44] J. B. Shearer, 'On a problem of Spencer', Combinatorica 5(3) (1985), 241-245.

[45] E. C. Titchmarsh, The Theory of Functions (Oxford University Press, 1939).

[46] S. Todo, 'Transfer-matrix study of negative-fugacity singularity of hard-core lattice gas', Internat. J. Modern Phys. C 10(4) (1999), 517-529. 